\title{
Phonetic variation in Italian consonant gemination
}

Elinor M. Payne

Department of Linguistics

University of Cambridge

emp14@cam.ac.uk

This paper presents findings on the articulatory and prosodic conditioning of Italian (lexical and post-lexical) geminate and non-geminate consonants. Phonetic and phonological prominence interact, resulting in a durational contrast which is not uniformly robust. So-called 'inherent' geminates are also found to be long post-consonantally, contrary to received wisdom, and do not form 'supergeminates' in conditions of postlexical gemination. Formant analysis of laterals reveals possible non-durational properties of gemination, with evidence for a more palatalised tongue configuration in geminates. Implications for the structural status of gemination are discussed.

\section{Introduction}

This paper presents the results of an investigation into durational and non-durational parameters of phonetic variability in Italian geminate and non-geminate consonants. By convention, geminates are characterised phonetically by increased duration. Durational variation in geminates has been investigated for various languages (e.g. Marotta 1986, Agostiniani 1992, Ericsdotter 1998 for Italian; Ladd \& Scobbie 2003 for Sardinian; Arvaniti 1999 for Cypriot Greek; Local \& Simpson 1999 for Malayalam; Louali \& Maddieson 1999 for Berber; Cohn, Ham \& Podesva 1999 for three Indonesian languages), and generally the duration contrast between geminates and non-geminates has been reported to be robust. However, previous studies have either focused on lexical geminates or have not tested all parameters of variation. Additionally, although most research has focused on the durational properties of geminates, some studies have also investigated non-durational indices. For example Local \& Simpson (1999) report the impression of a clear (more palatalised) resonance for geminate sonorants in Malayalam, and Abramson (1991, 1999) reports a greater intensity in the burst of geminate stops, and systematic differences in F0 in following vowels, for Pattani Malay.

The main aim of the present study is to compare different types of geminates and provide a more complete picture of the prosodic and articulatory conditioning of geminate duration and contrast for Italian. In particular, it seeks to establish whether the geminate contrast is uniformly robust, and how far consonant type, lexical stress and intonational 'prominence' shape variability in geminate duration and undermine the contrast with non-geminates. A further aim is to establish whether non-durational properties also distinguish geminates from non-geminates, specifically in gestural configuration, as possibly evident from formant structure. The two parts of the investigation are presented separately: the first part presents 
Table 1 Types of long consonant in Standard Italian.

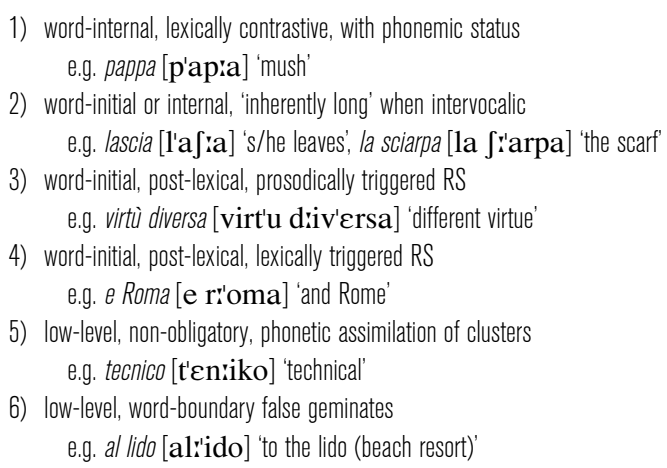

the results of an acoustic study of duration, while the second part presents an acoustic study of non-durational indices.

\section{Acoustic study of duration}

\subsection{Parameters of investigation}

\subsubsection{Geminate type}

In present-day Standard Italian, as spoken e.g. in Pisa (i.e. with minimal interference from other Italo-Romance dialects; for an overview of the complex linguistic situation in Italy see Maiden \& Parry 1997), there are arguably six types of long consonant, or geminate (see table 1). As a departure from convention, stress is marked immediately before the vowel, rather than at the beginning of the syllable. This is because geminate consonants are usually considered ambisyllabic, and marking stress before syllable-initial consonants would be problematic. Conventionally, Types 1,2,3 and 4 are classed as phonologically long consonants, or geminates, while Types 5 and 6 are classed as phonetically long consonants. Type 1 is lexically determined and contrasts phonemically with non-geminates (e.g. pappa vs. papa 'mush' vs. 'pope'). Type 2 consists of a set of consonants that are always phonetically long intervocalically and for which no contrasting non-geminate exists. They are conventionally classed also as phonologically long and it is assumed that the absence of a short (nongeminate) variant is the result of a defective paradigm. Types 3 and 4 are the result of a post-lexical process known as RADDOPPIAMENTO SINTATTICO (RS), or 'syntactic doubling'. In Type 4, certain lexical items (e.g. $e$ 'and' in table 1) will trigger gemination of the first consonant of the following word. Type 4 is present also in other central and southern dialects and varieties of Italian, though trigger words vary regionally. Type 3 is present only in certain central dialects and varieties of Italian. Marotta (1986) claims that prosodically triggered $\mathrm{RS}$ is a strategy for increasing the distance between primary stresses, and that the duration of the resulting geminate is inversely proportional to the size of interstress interval, i.e. /b:/ should be longer in città bella [t fit'a bíclia] than in città bellina [t fit:'a bicl'ina]. (Contrary to convention, in phonetic transcriptions lexical stress marks are placed just before the vowel rather than before the consonant. This is in order to avoid taking a position on syllabification of geminates, since placing the stress mark before a geminate consonant implies that geminate is tautosyllabic.) Observations that RS sometimes appears not to occur has led to claims (e.g. 
Table 2 Inherently long consonants. All examples are supposedly phonetically long, except for the post-consonantal context B.

\begin{tabular}{|c|c|c|}
\hline A lasciare & 'to leave' & 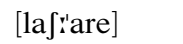 \\
\hline B non sciacqua & 's/he doesn't rinse' & [non S'ak:wa] \\
\hline C la sciacqua & 's/he rinses it' & [la 'frak:wa] \\
\hline D avrà sciarpe & 's/he will have scarves' & [avr'a fiarpe] \\
\hline
\end{tabular}

Nespor \& Vogel 1986) that higher-level boundaries may block its application, though other studies (e.g. Fanciullo 1986, Agostiniani 1992) refute this. For a more detailed description and historical account of RS, see Loporcaro (1997). Unlike Type 1 geminates, which in Standard Italian at least only appear word-internally, Type 2 geminates may also combine with an RS environment (either lexical or prosodic), e.g. e sciarpe 'and scarves'. In other words, Type 2 may interact with Types 3 and 4 . In this case, the geminate remains long, and it is assumed (though this has not been previously investigated) that no extra lengthening occurs.

Out of these six possible types, only four types were investigated: lexical (1); inherent (2); prosodically triggered RS (3) and false (6). Lexically determined RS (type 4) was not included because, being restricted to only a few trigger words, conditions could not be controlled. Low-level assimilatory geminates (type 5) were not included for similar reasons. The purpose of investigating different geminate types was to ascertain whether underlying structural differences are reflected in phonetic dissimilarity. Since consonant gemination word-internally (lexical geminates) can be the basis of a semantic contrast, while consonant gemination word-initially arises as a post-lexical process, it was hypothesised that word-internal (lexical) geminates would be consistently longer than word-initial (postlexical) geminates, and that the geminate/non-geminate contrast would also be greater in duration and less variable word-internally. A further hypothesis was that post-lexical geminates would be shorter than and pattern differently from false geminates. This hypothesis was based on the assumption that 'true' and false geminates are underlyingly different and that this is reflected in their phonetic interpretation. It is assumed that true geminates are underlyingly one consonant and involve one consonantal gesture, while false geminates are underlyingly and gesturally composed of two consonants. While the single consonant gesture of the true geminate is long, there is no need for it to be as long as twice the duration of a non-geminate.

\subsubsection{Inherently long consonants}

Like a handful of other consonants, / $/$ is supposedly (see Canepari 1999: 85; Rogers \& d'Arcangeli 2004: 117) long when intervocalic (see examples A and C in table 2) and short elsewhere (example B). Since $/ \int /$ can only be post-consonantal at word boundaries, geminate and non-geminate $/ \mathrm{J} /$ cannot be compared word-internally. It was hypothesised that intervocalic $/ \mathrm{f} /$ would be significantly longer than post-consonantal $/ \mathrm{f} /$. For the word-boundary condition, intervocalic $/ \mathrm{f} /$ in a $[-\mathrm{RS}]$ context (preceded by an unstressed vowel, example $\mathrm{C}$, supposedly already long because intervocalic) was compared with intervocalic $/ \mathrm{J} /$ in a $[+\mathrm{RS}]$ context (preceded by a stressed vowel, example D). It was hypothesised that an extra-long consonant, or 'super-geminate' would be created in the $[+\mathrm{RS}]$ context.

\subsubsection{Consonant type}

With one small qualification, all consonant phonemes in Italian can be geminate, $/ \mathrm{p}: \mathrm{b}: \mathrm{t}:$ d: k: g: tf: d3: s: f: v: n: m: l: r: $\int: K_{:}$j: ts: dz:/, and all except the last five, $/ \int: K:$ n: ts: $\mathrm{dz} /$, which are always geminate intervocalically, contrast phonemically with their singleton counterparts. The situation with regard to $/ \mathrm{s}$ s:/ is regionally complex. In northern varieties of Italian, intervocalic singleton /s/ is interpreted phonetically as the voiced [z] while geminate $/ \mathrm{s} / \mathrm{is}$ interpreted as voiceless (although many northern speakers do not produce geminates). 
In southern varieties of Italian, both singleton and geminate are phonetically interpreted as voiceless intervocalically. In both northern and southern varieties there are just two phonemes, distinguished only by length. In central varieties, however, and in the prescribed standard variety, /s/ also contrasts with /z/ word-internally when intervocalic, e.g. chiese [kjese] 'he asked' vs. chiese [kjeze] 'churches'.

In the present study, eight consonant types were examined: [p t b d m $\left.1 \mathrm{f} \int\right]$. This subset of consonants was chosen so as to explore the effect of a certain set of parameters while keeping the dataset to a manageable size: place of articulation (bilabial (or labio-dental) vs. coronal); manner of articulation (stop, fricative, nasal, lateral); and, for the stops, phonological voicing (voiced vs. voiceless). Several exclusions were made to keep the data set to a manageable size. For example, only two places of stop articulation and only one inherently long consonant were chosen, while affricates were not examined at all. /g: v:/ were excluded because very few tokens exist. For fricatives, /f $\mathrm{v} /$ were preferred over $/ \mathrm{s} \mathrm{z} /$ on account of the latters' defective paradigm. $/ \mathrm{r}$ / was not examined because its geminate counterpart introduces another dimension which may distort the picture on duration, in that there is also a non-quantitative contrast (tap vs. trill). The consonants appeared in or across actual words (depending on whether the target consonants were word-internal or word-boundary), and these target words are listed in the appendix. The vowel context, in the large majority of cases, was /aCa/. The three cases in which another vowel context was used, for reasons of lexical paucity, were lexical and $\mathrm{RS} / \mathrm{t} \mathrm{t} /$, for which the context $/ \mathrm{a}-\mathrm{o} /$ was used; false geminate /tt/ and lexical, $\mathrm{RS}$ and false geminate $/ \mathrm{d} \mathrm{d}: \mathrm{dd} /$, for which the context $/ \mathrm{a}-\mathrm{I} /$ was used.

The aim was to examine whether intrinsic durational properties, of the type explored by e.g. Lehiste (1970), condition the way a consonant is geminated, and in particular whether these properties result in asymmetries in the duration contrast. For example, are the geminate counterparts of intrinsically short singletons also relatively short? Mapping the synchronic phonetic 'success' of gemination as a function of consonant type may help determine whether RS is ever actually blocked, or if apparent lack of RS is in fact PHONETICALLY WEAK gemination. It was hypothesised that articulatory factors would result in variable durational contrast, and would also interfere with strategies for producing false geminates (i.e. whether they are articulated as one consonant or two).

\subsubsection{Prosodic factors}

Temporal properties and 'prominence' (loosely defined as elements in the speech chain that stand out in some way, e.g. through changes in pitch, duration and/or loudness, on account of their position in a given prosodic constituent) are intricately linked at more than one level in Italian. At the phonetic level, duration is an important cue for rhythmic prominence and the phonetic manifestation of phonological stress and the tonic accent of an intonational phrase. At a more abstract level, lexical stress is the trigger for prosodically triggered RS (type 3 geminates), and also played a role historically in the creation of new geminates word-internally (see e.g. Loporcaro 1997). Geminates may precede (fattóre) or follow (fátto) a main stress, or do neither (fattoríno), or indeed, at word boundaries, do both (l'avrà fátto). (Acute accents indicate lexical stress and do not reflect Italian orthography.) Phonetically, lexical stress may receive the greatest prominence in the intonational phrase, or be much reduced. Since lexical stress, which is a trigger for increased duration, interacts with intonational prominence at the phrase level, one objective of this study was to investigate how such interaction affects duration, and to investigate more fully the hypothesis (Marotta 1986) that RS is a strategy for avoiding stress-clash.

Consonants were tested in the NUCLEAR and a NON-NUCLEAR position of the intonational phrase. For nuclear position, the word or words in question fell at the end of the carrier sentence, e.g. il vento CALA, 'the wind drops', while for the non-nuclear position the word or words in question fell just before the end of the sentence, e.g. CALA il vento, 'drops the wind'. Because of the different types of geminate tested and the different conditions in which they appear (e.g. word-internal vs. word-boundary), it was not possible to have a consistent 
Table 3 Lexical stress conditions tested.

\begin{tabular}{|c|c|c|c|c|c|}
\hline \multicolumn{3}{|c|}{ Word-internal (WI) } & \multicolumn{3}{|c|}{ Word-boundary (WB) } \\
\hline & Non-geminate & Geminate & & Geminate & Non-geminate \\
\hline Pre-stress & VC'V & VCr'V & Pre-stress & 'V\#C!'V & V\#C'V \\
\hline Post-stress & 'VCV & 'VC:V & Pre-unstressed & 'V\#C:V(C'V) & V\#CV(C'V) \\
\hline Unstressed & VCV & VC:V & Min. stressed & 'V\#C:V(CV) & V\#CV(CV) \\
\hline
\end{tabular}

sentence frame without resorting to nonsensical sentences. However, efforts were made to keep to a consistent length (number of syllables) and a roughly equivalent syntactic structure.

Lexical stress conditions tested are set out in table 3. Consonants were in one of three positions: i) immediately before the main stress of the word (PRE-STRESS); ii) immediately after the main stress of the word (POST-STRESS) if word-internal, or before an unstressed syllable (PRE-UNSTRESSED) if word-boundary; or iii) between two unstressed vowels (UNSTRESSED), if word-internal or before two unstressed syllables (MINIMALLY STRESSED), if word-boundary. As can be seen from table 3, conditions could not be identical across the different types of geminate since at word boundaries post-stress consonants are necessarily geminate, whereas word-internally post-stress consonants can be geminate or non-geminate. The shaded boxes indicate those prosodic conditions that diverge from the 'norm'. For example, in the pre-stress condition, in only three out of four cases, the preceding syllable is unstressed: for wordboundary geminates, the preceding syllable is also, inevitably, stressed. It was hypothesised that gemination would be 'strongest' (duration longest and contrast with non-geminates greatest) when in close proximity to a lexical stress, and when that stress receives intonational prominence. It was also hypothesised that a post-stress position in particular would increase the duration of all consonants, on the basis that this is the trigger for gemination at word boundaries.

\subsection{Method}

The recordings were carried out in a sound-insulated room at the Linguistics Laboratory of the Scuola Normale Superiore in Pisa, Italy, using a high quality microphone and Sony DAT recorder. Five male speakers of Pisan Italian, aged between 19 and 38, read out four uniquely randomised repetitions of the entire set of carrier sentences (in which the relevant consonants were embedded) at a natural, conversational pace. The recordings were digitised at a sampling rate of $16 \mathrm{KHz}$ and analysed using Xwaves for Unix on a Silicon Graphics Indigo workstation at the University of Cambridge Phonetics Laboratory.

\subsection{Analysis}

Duration measurements were obtained through segmentation of the waveform, with reference to the spectrogram. The duration of consonants was taken to be the period of time elapsed between two points as indicated in table 4 . The decision to include VOT in the measurement of voiceless stops was based on perceptual considerations. From a production perspective, VOT arguably belongs both to the following vowel (supralaryngeally) and the consonant (laryngeally), but since it delays perception of the vowel, it was deemed to belong more to the consonant. A further practical consideration was the difficulty in separating the fricated release from aspiration.

Using the statistical package SuperANOVA, multivariate repeated measures ANOVAs (analysis of variance) were run on the duration measurements for each consonant. Statistical 
Table 4 Measurement of consonant duration.

\begin{tabular}{|c|c|c|}
\hline & From & To \\
\hline Voiceless stops & $\begin{array}{l}\text { Sudden reduction in amplitude and cessation of energy } \\
\text { in F2 and above }\end{array}$ & $\begin{array}{l}\text { Start of periodicity after release and } \\
\text { voice-delaying aspiration }\end{array}$ \\
\hline Voiced stops & $\begin{array}{l}\text { Sudden reduction in amplitude and attenuation of energy } \\
\text { in F2 and above }\end{array}$ & $\begin{array}{l}\text { Sudden rise in amplitude and in energy in F2 } \\
\text { and above after release }\end{array}$ \\
\hline Fricatives & Sudden reduction in amplitude and the start of aperiodic noise & Sudden rise in amplitude and start of periodicity \\
\hline Sonorants & Sudden reduction in amplitude and lowering of formants & Sudden rise in amplitude and formants \\
\hline
\end{tabular}

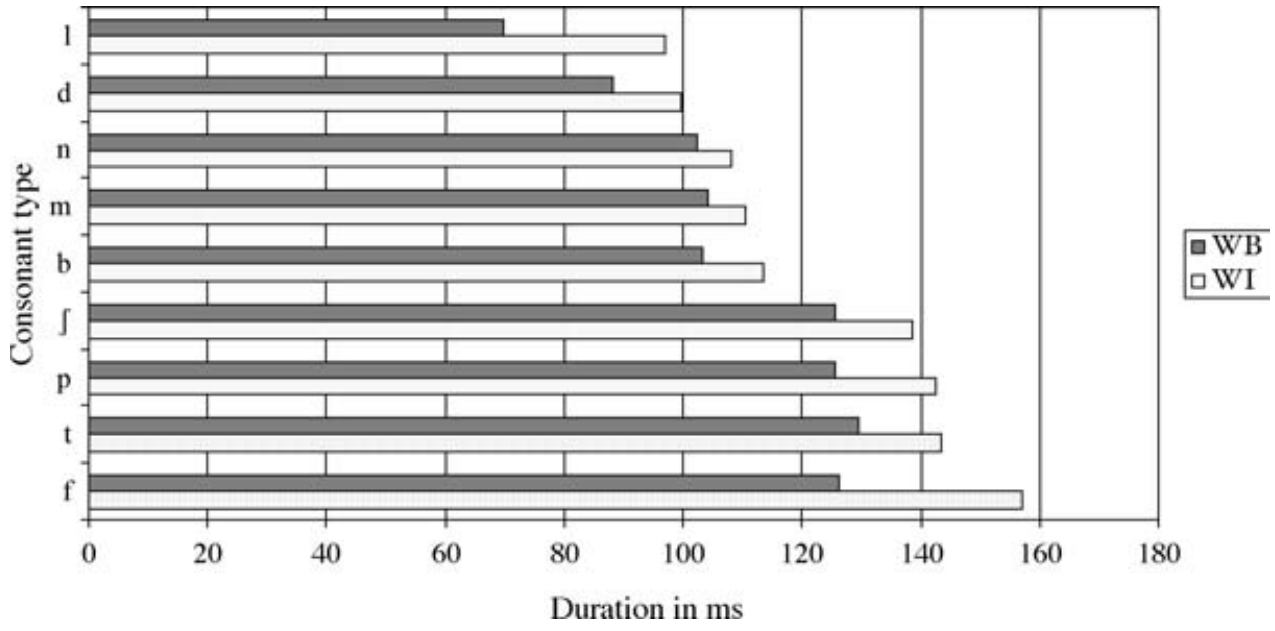

Figure 1 Mean duration of word-internal and word-boundary true geminates.

significance is taken to be attained when $\mathrm{P}<0.05$. Marginal statistical significance is taken to be attained when $\mathrm{P}>0.05$ but $<0.06$.

\subsection{Results}

What follows is a subset of findings presented in Payne (2000). Geminate duration is highly 'plastic', exhibiting systematic variation according to consonant type, geminate type and prosodic factors. Excluding false geminates, which will be discussed separately, the actual durations of individual tokens across consonant type range from $20.6 \mathrm{~ms}$ to $234.2 \mathrm{~ms}$ (range $=213.6 \mathrm{~ms}$; overall mean $=106.7 \mathrm{~ms}$ ), while mean durations (averaged across speakers) vary from $83.3 \mathrm{~ms}$ to $141.2 \mathrm{~ms}$, according to consonant type. Non-geminate duration also varies but to a lesser degree. Excluding / $/$, which will be discussed separately, actual non-geminate durations of individual tokens range from $9.4 \mathrm{~ms}$ to $134.9 \mathrm{~ms}$ (range $=125.5 \mathrm{~ms}$; overall mean $=61.45 \mathrm{~ms}$ ), while mean durations vary from $35.5 \mathrm{~ms}$ to $90.5 \mathrm{~ms}$.

\subsubsection{Consonant type}

Figures 1 and 2 show mean duration, across subjects, of true geminates and non-geminates according to consonant type and position in the word. As might be expected, voiceless consonants are longer than their voiced counterparts (on average and across type, 1.36 times for geminates and 1.6 times for non-geminates). Extremely short minima are obtained for $/ \mathrm{t} d /$ 
and especially $/ 1 /$, for which the minimum of $9.4 \mathrm{~ms}$ was measured. Such short durations suggest these consonants were occasionally produced as taps.

\subsubsection{Geminate type/position in the word}

Averaged over all prosodic conditions, lexical geminates are longer than post-lexical geminates. In other words, geminates in word-boundary position are always shorter than geminates in word-internal position. Geminate type proves to be a main effect for each consonant type except $/ \mathrm{m}:$ ( (see table 5 for results). However, analysis of individual prosodic conditions (lexical stress and intonational prominence) revealed that lexical geminates were not always longer than their post-lexical counterparts, and even where they were, the difference was not always statistically significant (see tables 6-8). Nevertheless, in nuclear position, lexical geminates were always longer than post-lexicals when post-stress/pre-unstressed, and this difference was always statistically significant (see table 6). This is noteworthy because it is uniquely in this condition that the prosodic environment is identical for both types of geminate (i.e. 'VC:V) and a thoroughly controlled comparison is possible. We can, therefore, conclude with reasonable confidence that for this stress condition, lexical geminates are longer than post-lexicals and reasonably suppose that the difference is due either to position in the word and/or geminate type.

Pre-stress (table 7), the trend is still for lexical geminates to be longer (with the exception of $/ \mathrm{b}: \mathrm{m}: /$ ), and this is significant (or marginally so, for $/ \mathrm{f}: /$ ). In unstressed condition (table 8), lexicals are longer than post-lexicals for all consonant types except $/ \mathrm{d} /$, , but the difference is only significant for $/ \mathrm{t}: \mathrm{f}: /$ (and marginally so for $/ \mathrm{b}: \mathrm{p}: /$ ).

In non-nuclear position, the picture is more erratic. The tendency in post-stress position (table 10) is again for geminates to be longer when lexical (with the exception of $/ \mathrm{t}: \mathrm{m}: /$ ), but this is only statistically significant for the lateral, /d: p: f: $1: /$ ). A similar pattern emerges in pre-stress position (table 9), with lexicals significantly longer only for /b: p: f: l:/ and postlexicals actually significantly longer for $/ \mathrm{d}$ : $\mathrm{t} /$. In unstressed position (table 11), lexicals are longer for /p: b: t: f: m:/, but only significantly so for $/ \mathrm{b}: \mathrm{f}: /$.

Non-geminate duration also varies according to position in the word (see figure 2), but not in a uniform manner: averaged over all prosodic conditions, $/ 1 \mathrm{~d} \mathrm{p} \mathrm{f/are} \mathrm{longer} \mathrm{word-}$ internally, while $/ \mathrm{m} \mathrm{b} \mathrm{t} /$ are longer at word boundaries. The differences are much smaller than for geminates (see table 12), but are nevertheless significant for $/ \mathrm{p} \mathrm{d} \mathrm{f} \mathrm{b/.} \mathrm{However,}$ further analysis reveals a strong tendency for word-internal non-geminates to be longer than their word-boundary equivalents only in pre-unstressed position, and typically only when nuclear. This trend is likely to result from the difference in lexical stress environment for nongeminates since, for this condition, word-internal non-geminates are preceded by a stressed vowel ('VCV), while word-boundary non-geminates are flanked by two unstressed vowels $(\mathrm{V} \# \mathrm{CV})$. For other prosodic conditions, which show an identical stress environment between word-internal and word-boundary, there is a greater tendency for word-boundary consonants to be longer, though the difference is seldom statistically significant.

In summary, position in the word is a significant factor for the duration of geminates where underlying structural differences are also at stake - but much less clearly so for nongeminates. Lexical geminates tend to be longer than post-lexical, and this is especially the case for i) post-stress/pre-unstressed position (all consonant types); ii) laterals and voiceless obstruents. Since Standard Italian does not have lexical geminates in word-initial position, we cannot know for sure whether the observed effect is a result of difference with respect to position in the word or the underlying structural difference in the two kinds of geminate. However, if word position were the cause, we would perhaps expect word-initial geminates to be longer, given a general universal tendency for consonants to be longer when word-initial (see e.g. Keating, Wright \& Zhang 1999), a tendency borne out, at least weakly, by certain non-geminates in the present study. 
Table 5 F ratios, significance levels, mean duration in ms and standard deviations for geminates (word-internal vs. word-boundary, across prosodic conditions). Each F: $(120,1)$.

\begin{tabular}{lcccl}
\hline & Word-internal duration (sd) & Word-boundary duration (sd) & $\mathrm{F}$ & $\mathrm{P}$ \\
\hline /b:/ & $111.340(23.18)$ & $102.396(23.774)$ & 18.17 & 0.013 \\
/p:/ & $143.120(32.961)$ & $126.625(21.883)$ & 63.467 & 0.0013 \\
$/ \mathrm{d}: /$ & $99.627(37.421)$ & $90.033(25.942)$ & 20.641 & 0.0105 \\
$/ \mathrm{t}: /$ & $143.270(33.883)$ & $129.750(27.187)$ & 34.543 & 0.0042 \\
$/ \mathrm{f}: /$ & $156.831(33.849)$ & $125.849(29.818)$ & 50.781 & 0.0021 \\
$/ \mathrm{l}: /$ & $96.883(34.631)$ & $6.694(22.005)$ & 461.554 & 0.0001 \\
$/ \mathrm{m}: /$ & $108.834(22.847)$ & $106.777(32.787)$ & 0.250 & 0.6433 \\
\hline
\end{tabular}

Table $6 \mathrm{~F}$ ratios, significance levels, mean duration in $\mathrm{ms}$ and standard deviations for geminates (nuclear post-stress/pre-unstressed word-internal and word-boundary). Each F: (20,1).

\begin{tabular}{|c|c|c|c|c|}
\hline & Word-internal duration (sd) & Word-boundary duration (sd) & $\mathrm{F}$ & P \\
\hline$/ \mathrm{b}: /$ & $146.336(14.937)$ & $102.835(9.72)$ & 92.305 & 0.0001 \\
\hline /p:/ & $186.553(14.877)$ & $149.36(17.198)$ & 79.031 & 0.0001 \\
\hline$/ \mathrm{d} \mathrm{s} /$ & $154.440(22.605)$ & $81.300(19.230)$ & 281.843 & 0.0001 \\
\hline$/ \mathrm{t}: /$ & $179.020(20.276)$ & $126.380(18.253)$ & 104.482 & 0.0001 \\
\hline$/ \mathrm{f}: /$ & $196.745(13.981)$ & $137.378(13.617)$ & 72.515 & 0.0001 \\
\hline /1:/ & 134.565 (15.571) & 64.565 (13.583) & 151.106 & 0.0001 \\
\hline$/ \mathrm{m}: /$ & $141.860(9.219)$ & $91.070(6.021)$ & 28.978 & 0.0007 \\
\hline
\end{tabular}

Table $7 \mathrm{~F}$ ratios, significance levels, mean duration in ms and standard deviations for geminates (nuclear pre-stress word-internal vs. word-boundary). Each F: $(20,1)$.

\begin{tabular}{lllrl}
\hline & Word-internal duration (sd) & Word-boundary duration (sd) & $\mathrm{F}$ & $\mathrm{P}$ \\
\hline /b:/ & $123.948(14.255)$ & $137.21(17.003)$ & 8.998 & 0.0171 \\
/p:/ & $170.638(22.295)$ & $137.311(24.582)$ & 60.694 & 0.0001 \\
/d:/ & $136.240(26.248)$ & $120.600(29.191)$ & 12.880 & 0.0071 \\
$/ \mathrm{t}: /$ & $182.060(30.328)$ & $164.740(26.000)$ & 11.311 & 0.0099 \\
$/ \mathrm{f}: /$ & $182.800(18.324)$ & $1666.897(19.006)$ & 5.204 & 0.0520 \\
$/ \mathrm{l}: /$ & $141.455(14.843)$ & $113.445(8.018)$ & 24.194 & 0.0012 \\
$/ \mathrm{m}: /$ & $130.850(9.627)$ & $135.940(6.850)$ & 0.291 & 0.6042 \\
\hline
\end{tabular}

\subsubsection{Prosodic factors}

Lexical stress is a main effect for consonant duration for all consonant types (see table 13) and intonational prominence (nuclear vs. non-nuclear position) is a main effect for all consonant types except $/ \mathrm{m} \mathrm{m:/} \mathrm{(see} \mathrm{table} \mathrm{14).} \mathrm{As} \mathrm{expected,} \mathrm{geminates} \mathrm{are,} \mathrm{on} \mathrm{average,} \mathrm{shorter} \mathrm{in} \mathrm{unstressed}$ conditions: averaged over all consonant types, unstressed geminates are respectively $80.72 \mathrm{~ms}$ and $76.89 \mathrm{~ms}$ shorter than post-stress and pre-stress geminates. Non-geminates are also mostly shorter in unstressed position (with the exception of $/ \mathrm{b} \mathrm{p} /$ which are, on average, shortest in pre-unstressed position), though the differences are smaller.

Again as expected, duration is, on average, longer in nuclear position, particularly for geminates (the mean difference for geminates over all consonant types is $36.37 \mathrm{~ms}$ ), though as further analysis reveals (table 15), it may be shorter in certain lexical stress conditions for 
Table 8 F ratios, significance levels, mean duration in ms and standard deviations for geminates (nuclear unstressed word-internal vs. word-boundary). Each F: $(20,1)$

\begin{tabular}{lcccc}
\hline & Word-internal duration (sd) & Word-boundary duration (sd) & $F$ & $P$ \\
\hline$/ \mathrm{b}: /$ & $100.031(8.52)$ & $89.639(11.212)$ & 5.309 & 0.0502 \\
$/ \mathrm{p} / /$ & $119.384(12.948)$ & $110.102(17.492)$ & 5.046 & 0.0549 \\
$/ \mathrm{d}: /$ & $79.720(10.718)$ & $88.360(17.366)$ & 3.933 & 0.0826 \\
$/ \mathrm{t}: /$ & $140.260(18.173)$ & $114.080(23.506)$ & 25.843 & 0.0009 \\
$/ \mathrm{f}: /$ & $140.045(19.431)$ & $100.455(13.057)$ & 32.249 & 0.0005 \\
$/ \mathrm{l} / \mathrm{l}$ & $70.745(9.565)$ & $63.345(10.392)$ & 1.689 & 0.2300 \\
$/ \mathrm{m}: /$ & $109.375(11.4)$ & $90.525(9.426)$ & 3.992 & 0.0808 \\
\hline
\end{tabular}

Table 9 F ratios, significance levels, mean duration in ms and standard deviations for geminates (non-nuclear pre-stressed word-internal vs. word-boundary). Each F: $(20,1)$

\begin{tabular}{lcccc}
\hline & Word-internal duration (sd) & Word-boundary duration (sd) & $F$ & $P$ \\
\hline$/ \mathrm{b}: /$ & $104.383(24.844)$ & $116.55(16.872)$ & 7.501 & 0.0255 \\
$/ \mathrm{p} / /$ & $128.845(22.749)$ & $118.736(81.732)$ & 5.485 & 0.0473 \\
$/ \mathrm{d}: /$ & $83.960(13.266)$ & $103.980(20.833)$ & 21.117 & 0.0018 \\
$/ \mathrm{t}: /$ & $127.280(16.654)$ & $141.040(25.977)$ & 7.139 & 0.0283 \\
$/ \mathrm{f}: /$ & $154.325(32.630)$ & $135.172(13.074)$ & 7.548 & 0.0252 \\
$/ \mathrm{l} / /$ & $95.665(20.146)$ & $63.625(5.604)$ & 31.657 & 0.0005 \\
$/ \mathrm{m}: /$ & $92.320(8.022)$ & $112.285(4.394)$ & 4.478 & 0.0672 \\
\hline
\end{tabular}

Table $10 \mathrm{~F}$ ratios, significance levels, mean duration in ms and standard deviations for geminates (non-nuclear post-stress word-internal vs. word-boundary). Each F: $(20,1)$.

\begin{tabular}{lcccc}
\hline & Word-internal duration (sd) & Word-boundary duration (sd) & $F$ & $P$ \\
\hline /b:/ & $92.451(15.239)$ & $86.329(15.934)$ & 1.816 & 0.2147 \\
/p:/ & $145.539(23.190)$ & $134.639(10.829)$ & 6.661 & 0.0326 \\
/d:/ & $75.600(12.076)$ & $62.100(13.864)$ & 9.602 & 0.0147 \\
$/ \mathrm{t}: /$ & $105.780(11.962)$ & $108.080(13.636)$ & 0.199 & 0.6670 \\
$/ \mathrm{f}: /$ & $144.380(33.498)$ & $126.123(16.252)$ & 6.858 & 0.0307 \\
$/ \mathrm{l}: /$ & $81.230(11.372)$ & $57.290(11.692)$ & 17.674 & 0.0030 \\
/m:/ & $88.185(11.481)$ & $93.415(9.822)$ & 0.307 & 0.5945 \\
\hline
\end{tabular}

some consonant types and position in the word. Nuclear geminates are longer for all stress conditions, irrespective of geminate type (position in the word) only for $/ \mathrm{b}: \mathrm{d}: \mathrm{l}: \mathrm{f:} /($ table 15 ), and these differences are not always statistically significant (see table 20), particularly for the unstressed condition. However, where duration is longer in NON-NUCLEAR geminates, the difference is not statistically significant.

As predicted, lexical stress and intonational prominence appear to interact. However, since lexical stress conditions vary between geminate and non-geminate and between wordinternal and word-boundary, analysis of this interaction is only meaningful for individual conditions. Tables 17-19 give the results of the statistical analysis for a comparison between 
Table 11 F ratios, significance levels, mean duration in ms and standard deviations for geminates (non-nuclear unstressed word-internal vs. word-boundary). Each F: $(20,1)$

\begin{tabular}{|c|c|c|c|c|}
\hline & Word-internal duration (sd) & Word-boundary duration (sd) & $\mathrm{F}$ & $P$ \\
\hline /bal/ & 99.932 (11.18) & $81.292(11.552)$ & 15.308 & 0.0045 \\
\hline /p:/ & $111.147(11.319)$ & $110.386(10.299)$ & 0.082 & 0.7816 \\
\hline /dis/ & $67.800(17.160)$ & $83.860(14.022)$ & 13.589 & 0.0062 \\
\hline$/ \mathrm{t}: /$ & $125.220(16.379)$ & $124.180(17.316)$ & 0.041 & 0.8450 \\
\hline$/ f: /$ & $122.690(19.127)$ & 87.428 (16.176) & 25.583 & 0.0010 \\
\hline /1:/ & $57.640(16.128)$ & $55.895(5.777)$ & 0.94 & 0.7671 \\
\hline$/ \mathrm{m}: /$ & $90.415(3.586)$ & $117.425(7.495)$ & 8.195 & 0.0211 \\
\hline
\end{tabular}

Table 12 F ratios, significance levels, mean duration in ms and standard deviations for non-geminates (non-nuclear unstressed word-internal vs. word-boundary). Each F: $(120,1)$.

\begin{tabular}{lllrl}
\hline & Word-internal duration (sd) & Word-boundary duration (sd) & $F$ & $P$ \\
\hline /b:/ & $53.64(12.948)$ & $59.39(13.442)$ & 9.583 & 0.0364 \\
/p:/ & $85.43(14.829)$ & $77.172(16.446)$ & 14.695 & 0.0186 \\
/d:/ & $55.733(8.325)$ & $48.827(10.146)$ & 10.699 & 0.0308 \\
/t:/ & $66.857(9.617)$ & $72.323(11.772)$ & 5.648 & 0.0763 \\
/f:/ & $99.391(12.466)$ & $81.604(20.742)$ & 16.445 & 0.0154 \\
/: $/$ & $35.706(10.207)$ & $35.301(9.966)$ & 0.102 & 0.7650 \\
/m:/ & $56.607(9.398)$ & $57.794(6.853)$ & 0.830 & 0.7872 \\
\hline
\end{tabular}

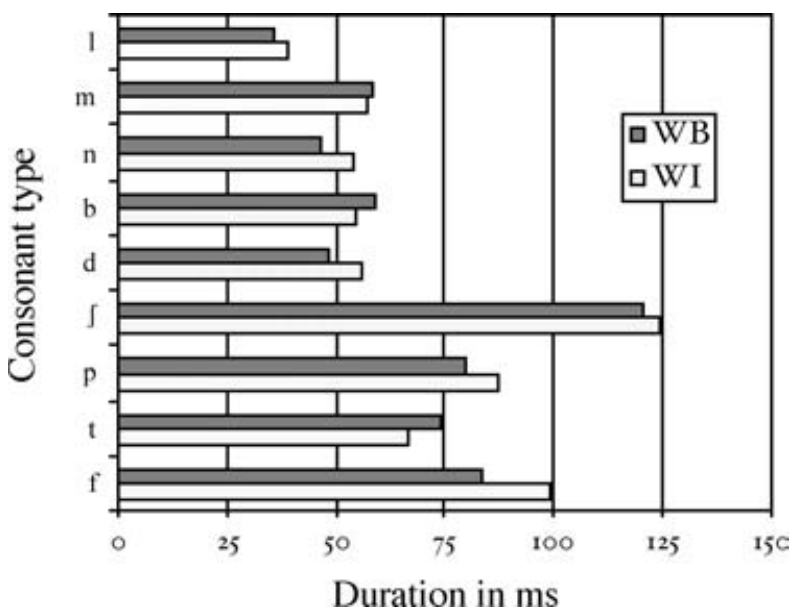

Figure 2 Mean duration of word-internal and word-boundary non-geminates.

lexical stress conditions. Word-internally, the influence of lexical stress on geminate duration depends on whether that stress is intonationally prominent (nuclear) or not (non-nuclear). Nuclear geminates are longest when POST-STRESS (except /l: t:/) (table 15), and the difference between post-stress and pre-stress is statistically significant (except for /f: m:/) (table 17) and that between post-stress and unstressed is statistically significant for all consonant types 
Table $13 \mathrm{~F}$ ratios, significance levels, mean duration in ms and standard deviations for geminates and non-geminates (comparing lexical stress conditions). Each F: (80, 2)

\begin{tabular}{lccccc}
\hline & Pre-stress duration (sd) & Post-stress duration (sd) & Unstressed duration (sd) & $F$ & $P$ \\
\hline$/ \mathrm{b}: /$ & $120.088(21.362)$ & $107.392(27.184)$ & $92.736(13.110)$ & 16.013 & 0.0016 \\
$/ \mathrm{b} /$ & $61.494(13.409)$ & $51.621(11.281)$ & $56.208(11.237)$ & & \\
$/ \mathrm{p}: /$ & $138.725(28.962)$ & $153.116(25.718)$ & $112.028(12.886)$ & 16.307 & 0.0015 \\
$/ \mathrm{p} /$ & $92.072(13.261)$ & $73.078(16.067)$ & $78.255(11.008)$ & & \\
$/ \mathrm{d}: /$ & $111.195(29.156)$ & $93.360(40.210)$ & $79.935(15.892)$ & 50.608 & 0.0001 \\
$/ \mathrm{d} /$ & $57.395(10.339)$ & $50.805(9.301)$ & $48.640(7.961)$ & & \\
$/ \mathrm{t} /$ & $153.780(31.717)$ & $129.815(33.801)$ & $125.935(19.935)$ & 13.328 & 0.0028 \\
$/ \mathrm{t} /$ & $75.725(8.974)$ & $67.045(12.623)$ & $66.000(8.740)$ & & \\
$/ \mathrm{f}: /$ & $159.798(26.960)$ & $151.157(33.852)$ & $112.655(26.039)$ & 132.526 & 0.0001 \\
$/ \mathrm{f} /$ & $99.517(17.211)$ & $91.579(18.017)$ & $80.397(18.126)$ & & \\
$/ \mathbf{l} /$ & $103.548(31.491)$ & $84.412(33.282)$ & $61.906(11.797)$ & 24.198 & 0.0004 \\
$/ \mathbf{l} /$ & $37.246(7.915)$ & $37.941(12.111)$ & $31.323(8.577)$ & & \\
$/ \mathrm{m}: /$ & $117.849(18.901)$ & $103.632(24.289)$ & $101.935(36.508)$ & 4.472 & 0.0497 \\
$/ \mathrm{m} /$ & $59.831(8.085)$ & $59.179(6.851)$ & $52.591(7.845)$ & & \\
\hline
\end{tabular}

Table 14 F ratios, significance levels, mean duration in ms and standard deviations for geminates and non-geminates (nuclear vs. non-nuclear conditions). Each F: (120, 1).

\begin{tabular}{lcccc}
\hline & Nuclear (sd) & Non-nuclear (sd) & $F$ & $P$ \\
\hline$/ \mathrm{b}: /$ & $96.118(19.281)$ & 57.723 & 0.0016 \\
$/ \mathrm{b} /$ & $116.282(24.002)$ & $55.370(13.881)$ & & \\
$/ \mathrm{p} / /$ & $56.770(12.297)$ & $124.001(20.845)$ & 17.667 & 0.0137 \\
$/ \mathrm{p} /$ & $145.728(32.028)$ & $78.725(17.028)$ & & \\
$/ \mathrm{d} / /$ & $84.018(14.227)$ & $79.550(19.671)$ & 58.410 & 0.0016 \\
$/ \mathrm{d} /$ & $110.110(35.368)$ & $50.703(9.273)$ & & \\
$/ \mathrm{t} /$ & $53.857(10.285)$ & $121.930(20.094)$ & 90.088 & 0.0007 \\
$/ \mathrm{t} /$ & $151.090(33.737)$ & $69.317(10.383)$ & & \\
$/ \mathrm{f}: /$ & $69.863(11.771)$ & $128.353(30.198)$ & 21.601 & 0.0097 \\
$/ \mathrm{f} /$ & $154.053(35.868)$ & $86.697(17.132)$ & & \\
$/ \mathrm{l} / /$ & $94.299(20.637)$ & $68.558(19.135)$ & 47.435 & 0.0023 \\
$/ 1 /$ & $98.020(5.366)$ & $31.609(8.166)$ & & \\
$/ \mathrm{m}: /$ & $39.398(10.273)$ & $99.007(30.409)$ & 5.179 & 0.0852 \\
$/ \mathrm{m} /$ & $116.603(22.678)$ & $30.409(9.488)$ & & \\
\hline
\end{tabular}

(table 19). When non-nuclear, however, geminates are longest when PRE-STRESS (except /pz/ which is longest when POST-STRESS), and the difference between pre-stress and post-stress is statistically significant for $/ \mathrm{l}: \mathrm{t}: \mathrm{b}: /$ (table 17) while that between pre-stress and unstressed is statistically significant for /d: f: l:/ (table 18). A similar interaction between lexical stress and intonational prominence is evident in word-internal non-geminates, although the interaction is weaker and differences are much smaller. When nuclear, more non-geminates $(/ \mathrm{t} 1 \mathrm{f} \mathrm{m} /)$ tend to be longest when POST-STRESS (table 16). Differences between post-stress and prestress are not, however, statistically significant (table 17), while those between post-stress and unstressed are only significant for / $\mathrm{l}$ / ( table 19). When non-nuclear, all consonants are longest PRE-STRESS, except /f 1/ (table 16) but the difference between pre-stress and post-stress is statistically significant only for / p l/ (table 16) while that between pre-stress and unstressed is 
Table 15 Mean duration in ms and standard deviations for nuclear and non-nuclear geminates under different lexical stress conditions.

\begin{tabular}{|c|c|c|c|c|c|c|c|}
\hline & & \multicolumn{3}{|c|}{ Word-internal } & \multicolumn{3}{|c|}{ Word-boundary } \\
\hline & & Pre-stress (sd) & Post-stress (sd) & Unstressed (sd) & Pre-stress (sd) & Pre-unstressed (sd) & Unstressed (sd) \\
\hline /b:/ & Nuclear & $123.176(14.593)$ & 146.485 (12.730) & 100.492 (8.008) & $137.422(17.025)$ & $102.548(9.025)$ & 89.825 (11.592) \\
\hline$/ \mathrm{b}: /$ & Non-nuclear & $104.962(24.682)$ & 92.125 (15.693) & 99.006 (11.628) & 116.025 (16.004) & 86.614 (15.841) & $81.020(11.410)$ \\
\hline /p:/ & Nuclear & 170.284 (22.018) & 186.472 (14.461) & $119.020(12.275)$ & 137.572 (24.627) & 149.025 (17.002) & $110.025(17.426)$ \\
\hline /p:/ & Non-nuclear & 128.002 (22.025) & $145.526(23.772)$ & 111.257 (11.012) & 118.261 (18.332) & $134.100(10.526)$ & $110.626(10.267)$ \\
\hline /dz/ & Nuclear & $136.240(26.248)$ & $154.440(22.605)$ & 79.720 (10.718) & 120.600 (29.191) & 81.300 (19.230) & $88.360(17.366)$ \\
\hline$/ \mathrm{d} z /$ & Non-nuclear & 83.960 (13.266) & 75.600 (12.076) & $67.800(17.160)$ & 103.980 (20.833) & $62.100(13.864)$ & $83.860(14.022)$ \\
\hline$/ \mathrm{t}: /$ & Nuclear & $182.060(30.328)$ & $179.020(20.276)$ & $140.260(18.173)$ & $164.740(26.000)$ & $126.380(18.253)$ & $114.080(23.506)$ \\
\hline$/ \mathrm{t}: /$ & Non-nuclear & $127.280(16.654)$ & $105.780(11.962)$ & $125.220(16.379)$ & $141.040(25.977)$ & 108.080 (13.636) & $124.180(17.316)$ \\
\hline$/ f: /$ & Nuclear & $182.800(18.324)$ & 196.745 (13.981) & $140.045(19.431)$ & 166.897 (19.006) & 137.378 (13.627) & $100.455(13.057)$ \\
\hline /f:/ & Non-nuclear & 154.325 (32.630) & $144.380(33.498)$ & $122.690(19.127)$ & 135.172 (13.074) & $126.123(16.252)$ & $87.428(16.176)$ \\
\hline$/ 1: /$ & Nuclear & 141.455 (14.843) & 134.565 (15.571) & $70.745(9.565)$ & 113.445 (8.018) & 64.565 (13.583) & 63.345 (10.392) \\
\hline /lis/ & Non-nuclear & 95.665 (20.146) & 81.230 (11.372) & 57.640 (16.128) & 63.625 (5.604) & $57.290(11.692)$ & 55.895 (5.777) \\
\hline$/ \mathrm{m}: /$ & Nuclear & $130.850(9.627)$ & $141.860(9.219)$ & $109.375(11.400)$ & $135.940(6.850)$ & $91.070(6.021)$ & $90.525(9.426)$ \\
\hline /m:/ & Non-nuclear & $92.320(8.022)$ & 88.185 (11.481) & $90.415(3.586)$ & 112.285 (4.393) & 93.415 (9.822) & $87.425(3.495)$ \\
\hline
\end{tabular}

Table 16 Mean duration in ms and standard deviations for nuclear and non-nuclear non-geminates under different lexical stress conditions.

\begin{tabular}{|c|c|c|c|c|c|c|c|}
\hline & & \multicolumn{3}{|c|}{ Word-internal } & \multicolumn{3}{|c|}{ Word-boundary } \\
\hline & & Pre-stress (sd) & Post-stress (sd) & Unstressed (sd) & Pre-stress (sd) & Pre-unstressed (sd) & Unstressed (sd) \\
\hline$/ \mathrm{b} /$ & Nuclear & $53.728(16.592)$ & 49.582 (11.190) & 53.007 (12.017) & 65.841 (7.041) & 56.014 (15.714) & 59.401 (7.014) \\
\hline$/ \mathrm{b} /$ & Non-nuclear & 58.714 (14.052) & 48.184 (7.052) & 54.552 (11.581) & $66.052(14.141)$ & 48.520 (10.184) & $58.471(16.456)$ \\
\hline$/ \mathrm{p} /$ & Nuclear & 99.828 (11.088) & $87.375(14.583)$ & 82.092 (7.002) & $92.236(9.688)$ & $71.280(14.251)$ & 74.625 (12.787) \\
\hline$/ \mathrm{p} /$ & Non-nuclear & 89.826 (19.098) & $75.256(15.047)$ & 78.672 (10.008) & 90.352 (14.673) & $58.672(8.275)$ & 77.047 (16.673) \\
\hline$/ \mathrm{d} /$ & Nuclear & $61.560(8.282)$ & $57.180(9.066)$ & $50.040(5.176)$ & 60.540 (11.389) & $47.840(9.771)$ & 45.980 (8.910) \\
\hline$/ \mathrm{d} /$ & Non-nuclear & 56.660 (11.508) & 55.620 (5.985) & $53.340(7.923)$ & $50.820(9.396)$ & $42.580(4.700)$ & $45.200(8.852)$ \\
\hline$/ \mathrm{t} /$ & Nuclear & $69.200(7.461)$ & 78.860 (8.342) & $59.820(4.774)$ & $81.140(9.480)$ & $66.240(15.956)$ & $63.920(8.043)$ \\
\hline$/ \mathrm{t} /$ & Non-nuclear & $72.480(5.856)$ & 55.960 (3.755) & $64.820(5.666)$ & 80.080 (8.714) & $67.120(9.471)$ & $75.440(8.775)$ \\
\hline$/ \mathrm{f} /$ & Nuclear & 101.725 (19.684) & $110.575(7.446)$ & 96.115 (9.906) & 112.517 (10.460) & 77.807 (14.914) & $67.055(11.310)$ \\
\hline$/ \mathrm{f} /$ & Non-nuclear & 94.985 (11.859) & 97.877 (9.873) & 95.070 (10.973) & 88.843 (19.597) & 80.057 (16.211) & 63.348 (7.102) \\
\hline$/ 1 /$ & Nuclear & $42.430(6.520)$ & $48.710(8.420)$ & $31.145(7.507)$ & 43.940 (5.163) & 36.340 (12.160) & 33.820 (11.809) \\
\hline$/ 1 /$ & Non-nuclear & 31.445 (2.364) & $33.270(8.213)$ & 27.235 (9.944) & $31.170(6.710)$ & $33.445(14.484)$ & 33.090 (4.708) \\
\hline$/ \mathrm{m} /$ & Nuclear & 58.745 (4.228) & $64.030(5.421)$ & $52.200(5.442)$ & 64.105 (4.649) & 58.200 (1.532) & $50.950(5.018)$ \\
\hline$/ \mathrm{m} /$ & Non-nuclear & $56.515(14.584)$ & $55.655(9.457)$ & 52.495 (11.843) & 59.960 (4.500) & 58.830 (7.449) & $54.720(9.382)$ \\
\hline
\end{tabular}

statistically significant only for $/ \mathrm{p} /$ (table 18 ). Since few differences prove to be statistically significant, it is difficult to draw any firm generalisations.

Any influence lexical stress may exert on the duration of word-boundary geminates is erratic, and only weakly supports Marotta's (1986) hypothesis of stress-clash avoidance. As Marotta found in her own study, word-boundary geminates are longest when pre-stress (i.e. sandwiched between two lexical stresses) (table 15) and this is true for all consonant types except non-nuclear /m:/ and nuclear and non-nuclear $/ \mathrm{p} /$. For nuclear position, the difference between pre-stress (interstress interval $=0$ syllables) and pre-unstressed (interstress interval $=1$ syllable) is statistically significant for all consonant types (table 17 ), while for non-nuclear position, the difference is only significant for $/ \mathrm{t}: \mathrm{d}: \mathrm{b} / /$. The strong version of Marotta's hypothesis, i.e. that geminate duration decreases as interstress interval increases, is 
Table 17 F ratios and significance levels of durational difference between pre-stress and post-stress (word-internal, word-boundary, nuclear, non-nuclear geminates and non-geminates). Each $\mathrm{F}:(20,1)$.

\begin{tabular}{|c|c|c|c|c|c|c|c|c|}
\hline & \multicolumn{4}{|c|}{ Word-internal } & \multicolumn{4}{|c|}{ Word-boundary } \\
\hline & \multicolumn{2}{|c|}{ Nuclear } & \multicolumn{2}{|c|}{ Non-nuclear } & \multicolumn{2}{|c|}{ Nuclear } & \multicolumn{2}{|c|}{ Non-nuclear } \\
\hline & $F$ & $P$ & $F$ & P & $F$ & $P$ & $F$ & $P$ \\
\hline$/ \mathrm{b}: /$ & 25.431 & 0.0010 & 6.358 & 0.0357 & 57.219 & 0.0001 & 43.665 & 0.0002 \\
\hline$/ \mathrm{b} /$ & 0.915 & 0.3669 & 4.354 & 0.0704 & 3.827 & 0.0862 & 14.635 & 0.0050 \\
\hline$/ \mathrm{p}: /$ & 14.988 & 0.0047 & 16.895 & 0.0034 & 7.685 & 0.0242 & 14.988 & 0.0047 \\
\hline$/ \mathrm{p} /$ & 8.224 & 0.0209 & 11.516 & 0.0095 & 24.708 & 0.0011 & 57.752 & 0.0001 \\
\hline$/ \mathrm{d}: /$ & 17.452 & 0.0031 & 3.682 & 0.0913 & 81.373 & 0.0001 & 92.408 & 0.0001 \\
\hline$/ \mathrm{d} /$ & 1.011 & 0.3442 & 0.057 & 0.8173 & 8.498 & 0.0194 & 3.577 & 0.0952 \\
\hline$/ \mathrm{t}: /$ & 0.348 & 0.5713 & 17.430 & 0.0031 & 55.484 & 0.0001 & 40.962 & 0.0002 \\
\hline$/ \mathrm{t} /$ & 3.519 & 0.0975 & 10.290 & 0.0125 & 8.371 & 0.0201 & 6.333 & 0.0360 \\
\hline /f:/ & 4.000 & 0.0805 & 2.035 & 0.1916 & 17.928 & 0.0029 & 1.685 & 0.2305 \\
\hline$/ \mathrm{f} /$ & 1.611 & 0.2400 & 0.172 & 0.6892 & 24.789 & 0.0011 & 1.589 & 0.2431 \\
\hline$/ 1: /$ & 1.464 & 0.2608 & 6.426 & 0.0350 & 73.680 & 0.0001 & 1.238 & 0.2982 \\
\hline$/ 1 /$ & 1.216 & 0.3022 & 0.103 & 0.7568 & 1.781 & 0.2187 & 0.160 & 0.7000 \\
\hline$/ \mathrm{m}: /$ & 1.362 & 0.2768 & 0.192 & 0.6728 & 22.617 & 0.0014 & 4.000 & 0.0805 \\
\hline$/ \mathrm{m} /$ & 0.314 & 0.5907 & 0.008 & 0.9296 & 0.392 & 0.5488 & 0.140 & 0.9076 \\
\hline
\end{tabular}

Table $18 \mathrm{~F}$ ratios and significance levels of durational difference between pre-stress and unstressed (word-internal, word-boundary, nuclear, non-nuclear geminates and non-geminates). Each F: $(20,1)$.

\begin{tabular}{|c|c|c|c|c|c|c|c|c|}
\hline & \multicolumn{4}{|c|}{ Word-internal } & \multicolumn{4}{|c|}{ Word-boundary } \\
\hline & \multicolumn{2}{|c|}{ Nuclear } & \multicolumn{2}{|c|}{ Non-nuclear } & \multicolumn{2}{|c|}{ Nuclear } & \multicolumn{2}{|c|}{ Non-nuclear } \\
\hline & $\mathrm{F}$ & P & $\mathrm{F}$ & P & $\mathrm{F}$ & P & $\mathrm{F}$ & $P$ \\
\hline /b:/ & 26.315 & 0.0009 & 1.278 & 0.2911 & 108.850 & 0.0001 & 60.555 & 0.0001 \\
\hline$/ \mathrm{b} /$ & 0.008 & 0.9329 & 0.612 & 0.4564 & 1.701 & 0.2284 & 2.875 & 0.1284 \\
\hline /p:/ & 147.381 & 0.0001 & 16.119 & 0.0039 & 43.504 & 0.0002 & 3.840 & 0.857 \\
\hline$/ \mathrm{p} /$ & 15.360 & 0.0044 & 6.910 & 0.0302 & 16.895 & 0.0034 & 9.067 & 0.0168 \\
\hline /d:/ & 168.307 & 0.0001 & 13.759 & 0.0060 & 54.763 & 0.0001 & 21.328 & 0.0017 \\
\hline$/ \mathrm{d} /$ & 6.992 & 0.0295 & 0.581 & 0.4679 & 11.169 & 0.0102 & 1.664 & 0.2331 \\
\hline$/ \mathrm{t}: /$ & 65.881 & 0.0001 & 0.160 & 0.6996 & 96.770 & 0.0001 & 10.718 & 0.0113 \\
\hline$/ \mathrm{t} /$ & 3.318 & 0.1060 & 2.212 & 0.1752 & 11.181 & 0.0102 & 0.812 & 0.3939 \\
\hline$/ f: /$ & 37.611 & 0.0003 & 20.591 & 0.0019 & 90.829 & 0.0001 & 46.899 & 0.0001 \\
\hline /f/ & 0.648 & 0.4442 & 0.1487 & 0.9906 & 42.524 & 0.0002 & 13.374 & 0.0064 \\
\hline$/ 1: /$ & 154.187 & 0.0001 & 44.589 & 0.0002 & 77.404 & 0.0001 & 1.843 & 0.2117 \\
\hline$/ 1 /$ & 3.927 & 0.0828 & 0.547 & 0.4808 & 3.158 & 0.1134 & 0.114 & 0.7447 \\
\hline$/ \mathrm{m}: /$ & 5.181 & 0.0524 & 0.410 & 0.8450 & 23.169 & 0.0013 & 0.297 & 0.6008 \\
\hline$/ \mathrm{m} /$ & 0.481 & 0.5075 & 0.182 & 0.6813 & 1.944 & 0.2007 & 0.308 & 0.5938 \\
\hline
\end{tabular}

not supported. Only nuclear /f: t: b:/ and non-nuclear /f: b:/ show this gradiency in duration as a function of interstress interval, and the differences are only statistically significant in nuclear position. Further evidence against Marotta's hypothesis comes from the fact that there is a strong tendency for non-geminates to be longest when pre-stress (even significantly so in some cases, see table 16) and since non-geminates cannot be preceded by an unstressed syllable at word boundaries, this cannot be the result of a stress clash. 
166 E. M. Payne

Table 19 F ratios and significance levels of durational difference between post-stress and unstressed (word-internal, word-boundary, nuclear, non-nuclear geminates and non-geminates). Each F: $(20,1)$.

\begin{tabular}{|c|c|c|c|c|c|c|c|c|}
\hline & \multicolumn{4}{|c|}{ Word-internal } & \multicolumn{4}{|c|}{ Word-boundary } \\
\hline & \multicolumn{2}{|c|}{ Nuclear } & \multicolumn{2}{|c|}{ Non-nuclear } & \multicolumn{2}{|c|}{ Nuclear } & \multicolumn{2}{|c|}{ Non-nuclear } \\
\hline & $F$ & $P$ & $\mathrm{~F}$ & $P$ & $F$ & $P$ & $F$ & $P$ \\
\hline /b:/ & 103.484 & 0.0001 & 1.935 & 0.2016 & 8.232 & 0.0345 & 1.378 & 0.2742 \\
\hline$/ \mathrm{b} /$ & 0.756 & 0.4099 & 1.701 & 0.2284 & 0.425 & 0.5326 & 4.538 & 0.0658 \\
\hline$/ \mathrm{pr} /$ & 256.368 & 0.0001 & 66.019 & 0.0001 & 87.758 & 0.0001 & 34.001 & 0.0004 \\
\hline$/ \mathrm{p} /$ & 1.106 & 0.3238 & 0.585 & 0.4664 & 0.740 & 0.4147 & 21.053 & 0.0018 \\
\hline$/ \mathrm{d} s /$ & 294.152 & 0.0001 & 3.205 & 0.1112 & 2.626 & 0.1438 & 24.947 & 0.0011 \\
\hline$/ \mathrm{d} /$ & 2.686 & 0.1399 & 0.274 & 0.6149 & 0.182 & 0.6807 & 0.362 & 0.5642 \\
\hline$/ \mathrm{t}: /$ & 56.647 & 0.0001 & 14.250 & 0.0054 & 5.705 & 0.0440 & 9.774 & 0.0141 \\
\hline$/ \mathrm{t} /$ & 13.669 & 0.0061 & 2.960 & 0.1237 & 0.203 & 0.6643 & 2.610 & 0.1448 \\
\hline$/ \mathrm{f}: /$ & 66.147 & 0.0001 & 9.680 & 0.0144 & 28.051 & 0.0007 & 30.807 & 0.0005 \\
\hline$/ \mathrm{f} /$ & 4.302 & 0.0718 & 0.162 & 0.6978 & 2.378 & 0.1616 & 5.744 & 0.0434 \\
\hline$/ 1: /$ & 125.603 & 0.0001 & 17.161 & 0.0032 & 0.046 & 0.8357 & 0.060 & 0.8126 \\
\hline$/ 1 /$ & 9.514 & 0.0150 & 1.123 & 0.3202 & 0.196 & 0.6698 & 0.004 & 0.9518 \\
\hline$/ \mathrm{m}: /$ & 11.854 & 0.0088 & 0.56 & 0.8191 & 0.003 & 0.9554 & 6.476 & 0.0677 \\
\hline$/ \mathrm{m} /$ & 1.572 & 0.2453 & 0.112 & 0.7463 & 0.590 & 0.4643 & 0.190 & 0.6746 \\
\hline
\end{tabular}

Table 20 F ratios and significance levels of durational difference between nuclear and non-nuclear (word-internal, word-boundary, geminates and non-geminates across lexical stress condition ( $\mathrm{i}=$ pre-stress, $i \mathrm{i}=$ post-stress/pre-unstressed, iii $=$ unstressed). Each $\mathrm{F}$ : $(20,1)$.

\begin{tabular}{|c|c|c|c|c|c|c|c|c|c|}
\hline & & \multicolumn{4}{|c|}{ Geminate } & \multicolumn{4}{|c|}{ Non-geminate } \\
\hline & & \multicolumn{2}{|c|}{ Word-internal } & \multicolumn{2}{|c|}{ Word-boundary } & \multicolumn{2}{|c|}{ Word-internal } & \multicolumn{2}{|c|}{ Word-boundary } \\
\hline & & $F$ & $P$ & $F$ & $P$ & $F$ & P & $F$ & $P$ \\
\hline$/ \mathrm{b}: \mathrm{b} /$ & i & 17.782 & 0.0028 & 20.050 & 0.0021 & 0.915 & 0.3669 & 0.017 & 0.8995 \\
\hline$/ \mathrm{b}: \mathrm{b} /$ & $\mathrm{ii}$ & 138.797 & 0.0001 & 12.400 & 0.0078 & 0.030 & 0.8663 & 3.024 & 0.1202 \\
\hline$/ \mathrm{b}: \mathrm{b} /$ & iii & 0.047 & 0.8334 & 3.334 & 0.1053 & 0.068 & 0.8008 & 0.068 & 0.8008 \\
\hline$/ \mathrm{p}: \mathrm{p} /$ & i & 101.704 & 0.0001 & 21.494 & 0.0017 & 5.263 & 0.0509 & 0.185 & 0.6784 \\
\hline$/ \mathrm{p}: \mathrm{p} /$ & $\mathrm{ii}$ & 96.941 & 0.0001 & 12.509 & 0.0070 & 7.952 & 0.0225 & 9.357 & 0.0156 \\
\hline$/ \mathrm{p}: \mathrm{p} /$ & iii & 3.840 & 0.0857 & 0.016 & 0.8998 & 1.007 & 0.3449 & 0.448 & 0.5222 \\
\hline$/ \mathrm{d}: \mathrm{d} /$ & $i$ & 144.002 & 0.0001 & 14.553 & 0.0051 & 1.265 & 0.2933 & 4.978 & 0.0562 \\
\hline$/ \mathrm{d}: \mathrm{d} /$ & $\mathrm{ii}$ & 327.484 & 0.0001 & 19.422 & 0.0023 & 0.128 & 0.7296 & 1.458 & 0.2618 \\
\hline /d: d/ & iii & 7.486 & 0.0256 & 1.067 & 0.3319 & 0.574 & 0.4705 & 0.032 & 0.8624 \\
\hline$/ \mathrm{t}: \mathrm{t} /$ & i & 113.150 & 0.0001 & 21.179 & 0.0018 & 0.406 & 0.5420 & 0.042 & 0.8421 \\
\hline$/ \mathrm{t}: \mathrm{t} /$ & $\mathrm{ii}$ & 202.259 & 0.0001 & 12.627 & 0.0075 & 19.773 & 0.0021 & 0.029 & 0.8686 \\
\hline$/ \mathrm{t}: \mathrm{t} /$ & iii & 8.529 & 0.0193 & 3.846 & 0.0855 & 0.943 & 0.3600 & 5.004 & 0.0557 \\
\hline$/ \mathrm{f}: \mathrm{f} /$ & i & 16.683 & 0.0035 & 20.708 & 0.0019 & 0.935 & 0.3620 & 11.531 & 0.0094 \\
\hline /f: f/ & $\mathrm{ii}$ & 56.419 & 0.0001 & 2.606 & 0.1451 & 3.318 & 0.1060 & 0.104 & 0.7552 \\
\hline /f: f/ & iii & 6.197 & 0.0376 & 3.491 & 0.0986 & 0.022 & 0.8846 & 0.283 & 0.6094 \\
\hline /1: $1 /$ & $i$ & 64.659 & 0.0001 & 76.541 & 0.0001 & 3.721 & 0.0899 & 5.029 & 0.0552 \\
\hline /1: $1 /$ & $\mathrm{ii}$ & 87.722 & 0.0001 & 1.632 & 0.2372 & 7.352 & 0.0266 & 0.258 & 0.6249 \\
\hline /1: $1 /$ & iii & 5.296 & 0.0504 & 1.712 & 0.2271 & 0.471 & 0.5117 & 0.016 & 0.9012 \\
\hline$/ \mathrm{m}: \mathrm{m} /$ & $i$ & 16.677 & 0.0035 & 6.286 & 0.0365 & 0.056 & 0.8191 & 0.193 & 0.6721 \\
\hline$/ \mathrm{m}: \mathrm{m} /$ & $\mathrm{ii}$ & 32.364 & 0.0005 & 0.062 & 0.8100 & 0.788 & 0.4006 & 0.004 & 0.9484 \\
\hline$/ \mathrm{m}: \mathrm{m} /$ & iii & 4.038 & 0.0793 & 8.129 & 0.0214 & 0.001 & 0.9758 & 0.160 & 0.6999 \\
\hline
\end{tabular}




\subsubsection{Geminate/non-geminate contrast}

The conventional view is that duration difference, both absolute and proportional, is robustly maintained between geminate and non-geminate in Italian (e.g. Ericsdotter 1998). The JND (Just Noticeable Difference) for segment duration lies in the region of 10-40 ms (Lehiste 1970: 13; Klatt 1976). A difference of $25 \mathrm{~ms}$, in the middle of this region, was taken as the threshold for consideration of the present study. The results of the present experiment show that, on average, geminates are 1.56 times longer than their non-geminate counterparts and that mean differences are over $25 \mathrm{~ms}$, providing further evidence of a robust distinction. (The one exception is 'geminate' $/ \mathrm{f} /$, which is only 1.11 times longer than 'non-geminate' $/ \int /$.) However, being means, such results smooth over any variation induced by particular prosodic and articulatory factors. Furthermore, the difference in plasticity of duration between geminates and non-geminates means that the duration contrast is non-uniform and can even be neutralised. Closer inspection reveals that consonant type, intonational prominence and proximity to lexical stress can conspire to undermine the durational contrast. The impact on the perception of difference may be buffered by durational cues from preceding vowels, with vowels being shorter before a geminate than before a non-geminate. However, this is only true of vowels in stressed syllables (Rogers \& d'Arcangeli 2004), precisely that context which favours the geminate contrast in consonants anyway.

There are three ways in which the durational contrast between geminate and non-geminate can potentially be undermined.

i) ACTUAL OVERLAP OF MINIMUM GEMINATE AND MAXIMUM NON-GEMINATE DURATION. Actual overlap is defined as occurring when the minimum duration of a geminate is at least $25 \mathrm{~ms}$ shorter than the maximum duration of its counterpart non-geminate for the same prosodic conditions. As table 21 shows, overlap is the norm here in non-nuclear position, while in nuclear position overlap is more common at word boundaries and in unstressed conditions.

Table 21 Actual duration overlap.

\begin{tabular}{lll}
\hline OVERLAP & Word-internal & Word-boundary \\
\hline Nuclear & Unstressed /f p l/ & Minimally stressed: ALL \\
& & Pre-unstressed: ALL except /p/ \\
& Pre-stress: only /p/ \\
Non-nuclear & ALL & ALL \\
\hline
\end{tabular}

ii) Taking a perceptual threshold for durational differences of $25 \mathrm{~ms}$, where the mean duration of a geminate (across speakers) is within $25 \mathrm{~ms}$ of the mean duration of its counterpart non-geminate, these might be considered cases of POTENTIAL PERCEPTUAL OVERLAP. As table 22 shows, potential perceptual overlap principally affects coronal sonorants and voiced stops.

Table 22 Potential perceptual overlap of mean durations.

\begin{tabular}{lll}
\hline OVERLAP & Word-internal & Word-boundary \\
\hline Nuclear & Unstressed /d/ & $\begin{array}{l}\text { Pre-unstressed /1/ } \\
\text { Minimally stressed /1/ }\end{array}$ \\
Non-nuclear & $/ \mathrm{d} /$ & Pre-stress /p/ \\
& Unstressed /f/ & Pre-unstressed /d l/ \\
& & Minimally stressed /f b m l/
\end{tabular}


iii) Differences below $25 \mathrm{~ms}$ between minimum actual geminate duration and maximum actual non-geminate duration might be considered cases of WEAK POTENTIAL PERCEPTUAL. This is the least likely scenario for perceptual confusion, since it does not take into account individual variation. As table 23 shows, overlap would appear to be possible for almost all consonants in all conditions. The one conspicuous absence from the table is word-internal nuclear post-stress, where there appears to be no risk of perceptual overlap in any consonant, suggesting a particularly robust distinction for this condition.

Table 23 Weak potential perceptual overlap.

\begin{tabular}{lll}
\hline OVERLAP & Word-internal & Word-boundary \\
\hline \multirow{2}{*}{ Nuclear } & Pre-stress: ALL except $/ \mathrm{t} 1 \mathrm{~m} /$ & ALL except $/ \mathrm{m} /$ \\
& Unstressed: ALL & ALL \\
\hline \multirow{2}{*}{ Non-nuclear } & ALL & \\
\hline
\end{tabular}

\subsubsection{Proportionate durations}

An alternative way of quantifying the contrast between geminates and non-geminates is to calculate the ratio of durations. This ratio is consistently greater word-internally (mean 1.9) than at word boundaries (mean 1.7). Within the word-internal context, the ratio is consistently greater when nuclear than when non-nuclear, and particularly high when adjacent to a stressed syllable. As figure 3 shows, in a nuclear context, values are higher pre-stress for $/ \mathrm{t} 1 /$, higher for post-stress for $/ \mathrm{p} \mathrm{d} \mathrm{b} \mathrm{J/} \mathrm{and} \mathrm{equally} \mathrm{high} \mathrm{for} \mathrm{pre-stress} \mathrm{and} \mathrm{post-stress} \mathrm{for} / \mathrm{m} \mathrm{f} /$, and smallest when unstressed for all consonant types. In a non-nuclear context, the pattern is more erratic, although the ratios are more commonly higher pre-stress (/f b d l/), and only higher post-stress for $/ \mathrm{p} /$.

Figure 4 shows geminate:non-geminate values (G:NG) for word-boundary consonants. The ratio is still generally higher when nuclear, but not for all consonant types and stress conditions. The highest nuclear values occur pre-stress for /t d b m 1/ and pre-unstressed

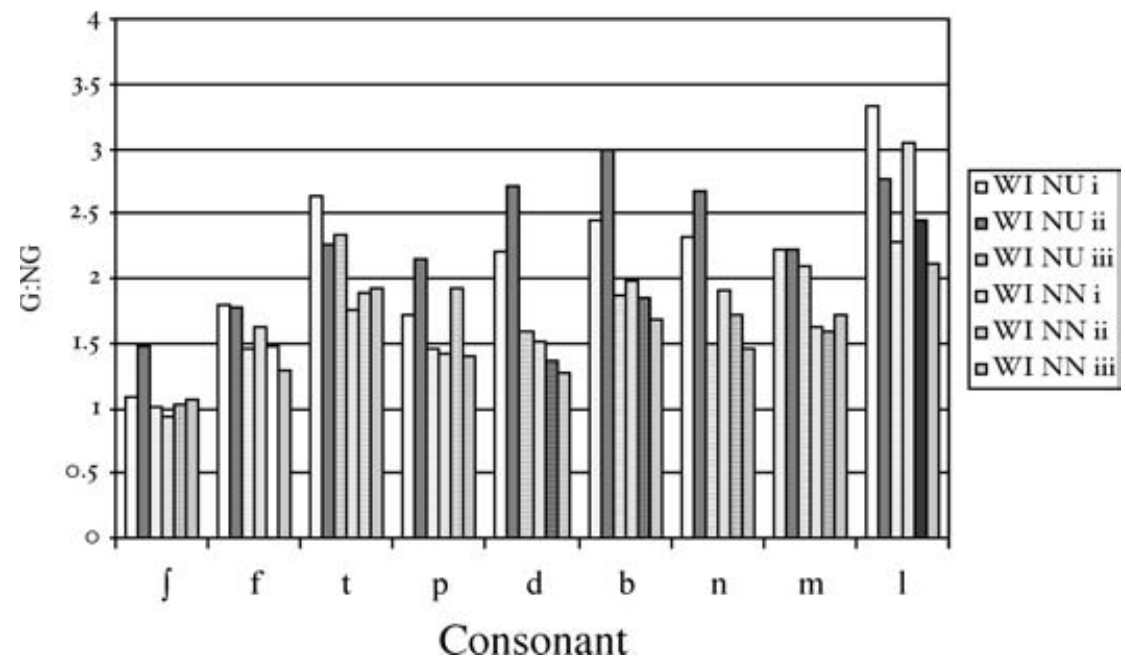

Figure 3 Geminate:non-geminate values for word-internal consonants as a function of stress and phrase position ( $\mathrm{i}=$ pre-stress, $\mathrm{ii}=$ post-stress, $i i \mathrm{i}=$ unstressed). 


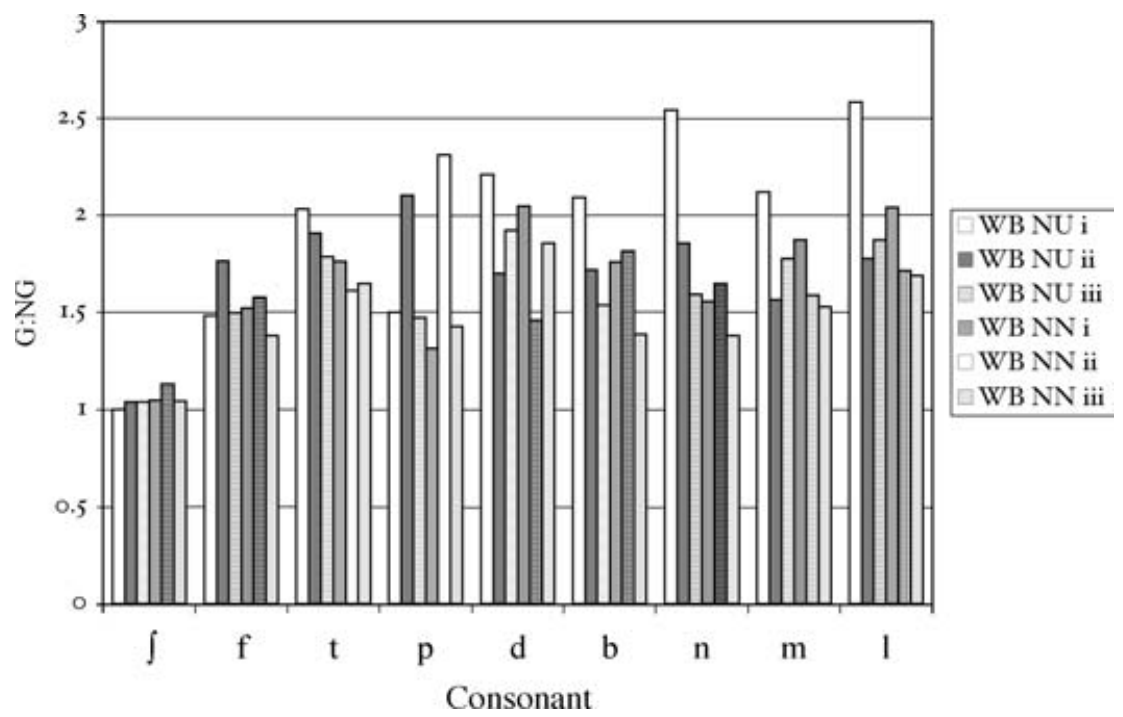

Figure 4 Geminate non-geminate values for word-boundary consonants as a function of stress and phrase position ( $\mathrm{i}=$ pre-stress, $\mathrm{ii}=$ post-stress, $\mathrm{iii}=$ unstressed).

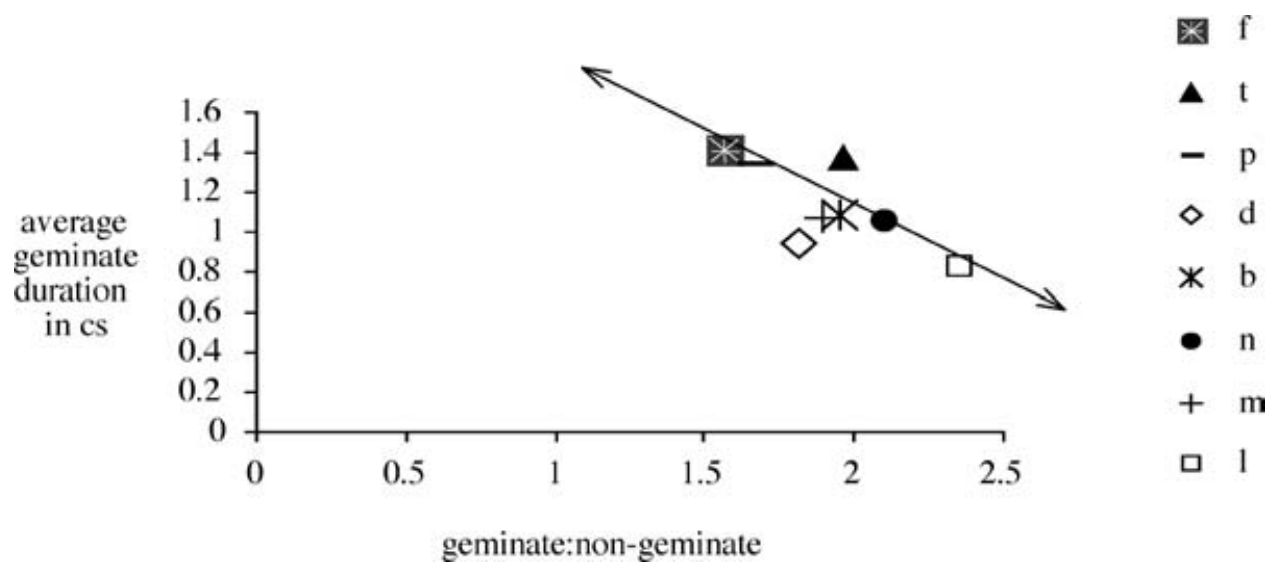

Figure 5 Geminate duration:non-geminate duration against average geminate duration.

for /f $\mathrm{p}$ / in a nuclear context, and pre-stress for $/ \mathrm{t} \mathrm{d} \mathrm{m} \mathrm{l} /$ and pre-unstressed for $/ \mathrm{f} \mathrm{p} \mathrm{b} /$ in a non-nuclear context.

G:NG were found to be in a roughly inverse relationship with mean absolute durations for geminates (see figure 5). Thus, /1/ has the shortest mean geminate duration but has the greatest G:NG, while /f $\mathrm{p}$ / have the longest mean geminate durations but the smallest G:NG. Interestingly, $/ \mathrm{l}: /$ is the joint most common lexical geminate, making up over $20 \%$ of the total number of geminates appearing in a corpus of over 40,000 words (Bortolini \& Zampolli 1979). Although the durations of /p: f:/ are very long, so, comparatively, are the durations of their non-geminate counterparts, and though the mean absolute differences are above the perceptual threshold, the proportional differences are very small. /p: f:/ also happen to be uncommon as lexical geminates $(2.43 \%$ and $1.71 \%$ of the total, respectively). G:NG for $/ \mathrm{b}$ : $\mathrm{m}: \mathrm{d}: /$ are moderately high but their absolute durations are very short, and their functional 
Table 24 Mean durations in ms and standard deviations for nuclear and non-nuclear word-internal and word-boundary / $/$ / across for stress conditions

\begin{tabular}{llllll}
\hline & & $\begin{array}{l}\text { Word-internal } \\
\text { (intervocalic) (sd) }\end{array}$ & $\begin{array}{l}\text { Word-boundary } \\
\text { (intervocalic) (sd) }\end{array}$ & $\begin{array}{l}\text { Word-boundary } \\
\text { (post-consonantal) (sd) }\end{array}$ & $\begin{array}{l}\text { Word-boundary } \\
\text { (RS condition) (sd) }\end{array}$ \\
\hline \multirow{2}{*}{ Nuclear } & Pre-stress & $167.947(27.717)$ & $162.512(27.233)$ & $156.562(27.030)$ & $158.260(29.261)$ \\
& Post-stress/pre-unstressed & $182.148(22.762)$ & $122.915(19.792)$ & $113.127(23.313)$ & $128.248(15.213)$ \\
\multirow{4}{*}{ Non-nuclear } & $123.925(20.766)$ & $105.605(18.311)$ & $122.153(20.422)$ & $110.872(16.371)$ \\
& Unstressed & $136.342(17.941)$ & $133.823(21.384)$ & $145.223(18.755)$ & $141.915(24.063)$ \\
& Pre-stress & $112.030(17.598)$ & $107.787(18.212)$ & $109.438(19.326)$ & $118.212(25.701)$ \\
& Post-stress/pre-unstressed & $113.628(19.351)$ & $105.218(19.248)$ & $107.078(16.964)$ & $108.462(18.031)$ \\
\hline
\end{tabular}

loads are low $(2.36 \%, 2.1 \%$ and $0.54 \%$ of the total respectively). $/ \mathrm{t} /$, however, which also has a moderately high G:NG, does have a very long absolute geminate duration, and has the joint highest functional load ( $20 \%$ of total). It would appear that the functional 'success' as a geminate of a particular consonant type is not necessarily 'disadvantaged' by a short geminate duration, as shown by the high functional load of $/ 1 /$. Similarly, a very long absolute geminate duration is not necessarily an advantage, as shown by the low functional loads of $/ \mathrm{p}: \mathrm{f}: /$. A high G:NG is critical, then, but unless accompanied by a long absolute geminate duration, it must be very high.

\subsubsection{Inherent geminates}

Word-internally, intervocalic / $/$ / is, as phonologists predict (Rogers \& d'Arcangeli 2004: 117), as long as other voiceless obstruent geminates. However, the word-boundary, supposedly nongeminate, post-consonantal $/ \mathrm{J} /$ is only shorter than its intervocalic counterpart (see table 24 ) when pre-stress or pre-unstressed in nuclear position. Even then, the difference is small and is only statistically significant for pre-unstressed condition $(\mathrm{F}(20,1)=5.674, \mathrm{P}=0.0444)$. The post-consonantal variant is LONGER for all other conditions and the difference is even statistically significant for nuclear unstressed $(\mathrm{F}(20,1)=16.217, \mathrm{P}=0.0038)$ and non-nuclear pre-stress $(\mathrm{F}(20,1)=7.696, \mathrm{P}=0.0241)$. Geminates overlap consistently with supposed nongeminates by around $50 \mathrm{~ms}$, irrespective of prosodic conditioning. The only exception is for nuclear post-stress/pre-unstressed position, when the word-internal 'geminate' is on average $56 \mathrm{~ms}$, or 1.47 times, longer than the 'non-geminate', and little overlap occurs. However, it should be recalled that stress conditions for the geminate and non-geminate are different in this condition. The 'non-geminate' in this case is actually word-boundary and not post-stress, and so the increased duration difference may be a result of stress-adjacency in the geminate coupled with non-stress adjacency in the 'non-geminate'. The results strongly suggest that, contrary to received wisdom, there is no phonetic evidence for a structural difference, and virtually no allophonic difference between intervocalic and post-consonantal $/ \mathrm{f} /$.

It is of note, however, that for word-internal consonants, post-stress position yields the longest consonants when in a nuclear context, while pre-stress positions yields the longest consonants in a non-nuclear context. The difference between nuclear pre-stress and post-stress is statistically significant $(\mathrm{F}(20,1)=11.943, \mathrm{P}=0.0086)$, as is that between post-stress and unstressed $(\mathrm{F}(20,1)=200.745, \mathrm{P}=0.0001)$. For non-nuclear position, the differences between pre-stress and the other stress conditions are statistically significant $(\mathrm{F}(20,1)=35.001, \mathrm{P}=0.0004$ and $\mathrm{F}(20,1)=30.550, \mathrm{P}=0.0006$, respectively $)$, while that between the other two conditions is not $(\mathrm{F}(20,1)=0.151, \mathrm{P}=0.7075)$. This follows the same pattern as other consonant types.

Given that duration in $/ \mathrm{J} /$ is never contrastive, it is of little consequence whether it is represented phonologically as geminate or non-geminate, though the fact that $/ \mathrm{J} /$ is always 


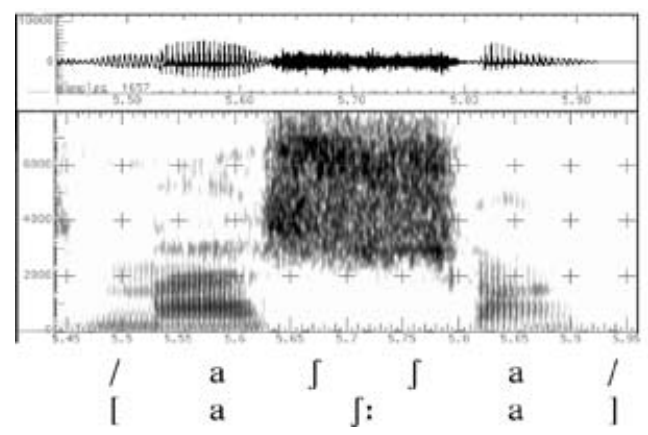

Figure 6a Articulatory 'fusion'.

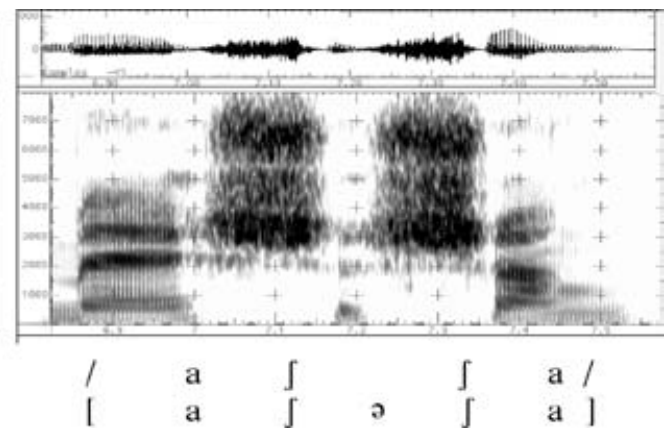

Figure 6b Separate articulation.

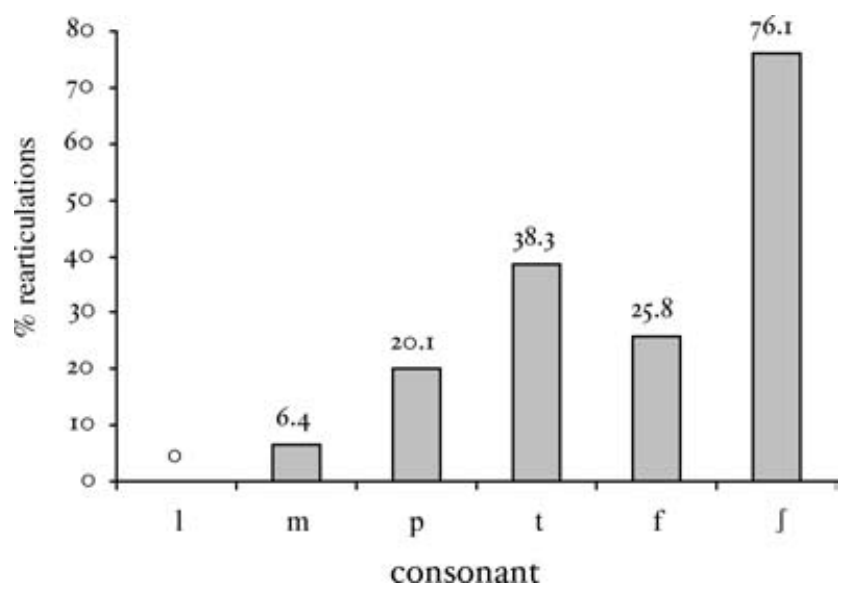

Figure $7 \%$ rearticulations in false geminates.

phonetically long means it is perhaps more appropriate to represent it as geminate, and assume that there is a gap in the non-geminate inventory. At word boundaries, a [+RS] context increases the mean duration of $/ \mathrm{J} /$, across prosodic conditions, by only $9 \mathrm{~ms}$. The only condition under which the difference is statistically significant is non-nuclear pre-unstress $(\mathrm{F}(20,1)=6.436 ; \mathrm{P}=0.349)$. The evidence for the creation of a 'super-geminate' is, therefore, extremely weak, and it is possibly because $/ \mathrm{J} /$ has already reached its upper durational limit. This supports the claim that $/ \mathrm{J} /$ has special status as inherently geminate and is not affected by RS.

\subsubsection{False geminates}

As with true geminates, the duration of false geminates varies according to consonant type, i.e. sonorants are shorter than obstruents. $/ \int \mathrm{f} /$, which are phonetically longer than other consonants when non-geminate, are also particularly long as false geminates. The articulation of false geminates varies: either there is articulatory 'fusion' (figure 6a), resulting in a shorter overall duration, or both elements are articulated, usually separated by an epenthetic vowel (figure 6b).

Sonorants 'fuse' more freely than obstruents (see graph in figure 7), with false geminate /11/ consistently fused. Susceptibility to 'fusion' in false geminates probably reflects phonotactic constraints. In Italian, the only consonants which can occur word-finally in 
indigenous, non-learned words are the sonorants $/ 1 \mathrm{n} \mathrm{r}$, and even these tend only to be word-final in certain grammatical words (e.g. il, non, per 'the', 'not', 'for') which do not, ordinarily, occur phrase-finally. The phonetic factors which underlie phonotactic constraints may be gradient, but the structure they establish is categorical. The sonorant $/ \mathrm{m} /$ cannot appear word-finally in Italian and thus would appear to lie on the wrong side of a categorical threshold.

On average, false geminates are longer than true geminates, though the difference decreases in magnitude as susceptibility to fusion increases, to the point that there is virtually no difference between false and true geminate $/ \mathrm{l} /$. Both are also marginally longer than twice the duration of non-geminate $/ 1 /$. False geminates (except $/ \mathrm{f} /$ ) pattern more like non-geminates in that they are longest pre-stress when nuclear; voiceless obstruents are also longest pre-stress when non-nuclear, while sonorants are longer when pre-unstressed.

\subsection{Discussion: duration and contrast}

It has been shown that geminate and non-geminate duration, and the contrast between them, vary according to consonant type, position in the word (or geminate type), and word- and phrase-level prosodic factors. On consonant type, each consonant appears to have an inbuilt relationship between geminate and non-geminate duration and there is an apparent association between this relationship and a geminate's functional load, with the result that $/ \mathrm{t}: \mathrm{l} / \mathrm{are}$ particularly 'successful' geminates. On position in the word, lexical geminates are longer than post-lexical geminates, especially in a post-stress/pre-unstressed position, and particularly for laterals and voiceless obstruents. This is more likely to be an effect of geminate type, since non-geminates pattern, albeit weakly, in the opposite manner. This reverse trend in non-geminate behaviour also contributes to the greater robustness of the durational contrast word-internally, with fewer incidences of actual or potential overlap occurring, and a consistently greater G:NG.

Both phonological prominence (adjacency to lexical stress) and phonetic prominence (determined by position in the intonational phrase) increase phonetic duration, particularly in geminates, and decrease the likelihood of overlap between geminates and non-geminates, thereby also increasing the robustness of contrast. In conditions of very low prominence (phonetic and/or phonological), the duration contrast is much reduced, and sometimes neutralised. Consonant gemination in Italian can therefore be thought of as intrinsically linked to PROMINENCE and, like prominence, is arguably both a phonetic and phonological event.

The direction of influence which prominence exerts is also of interest. Post-stress position is uncontroversially a critical factor at word boundaries, since this is the conditioning environment for (prosodically triggered) RS. However, in NUCLEAR position, post-stress position also causes consonant lengthening, albeit at the phonetic level, WORD-INTERNALLY. Though this is most clearly true for geminates, there is a similar, if albeit weaker and not statistically significant, trend also in non-geminates. Moreover, the proportional duration of geminates is greater post-stress, for most consonant types. Thus, post-stress position not only causes consonant lengthening, it is also the optimal position for establishing a duration distinction. However, in a NON-NUCLEAR context, it is more common for geminates and nongeminates to be longer, and for the proportional duration of geminates to be greater, when PRESTRESS. Thus, post-stress position produces phonetic lengthening so long as is it coincident with phonetic prominence. When this is not the case, pre-stress position has a greater effect on consonant duration. In other words, the domain of influence exerted by phonological prominence extends regressively unless that prominence is rendered phonetically prominent by position in the phrase and semantically driven intonational patterns, in which case it extends progressively. This pattern of behaviour is shown in figure 8 .

The patchy robustness of the duration contrast raises some interesting questions. Where phonetic signalling of contrast is unreliable, semantic context can be presumed to play a critical role, but this is really only necessary in the case of lexical geminates. There is no a priori reason 


\begin{tabular}{l|cc} 
Intonational prominence & Pre-nuclear & Nuclear \\
\hline Lexical stress & VC"V & "VCV \\
\hline $\begin{array}{l}\text { Direction of influence } \\
\text { (lengthening effect on consonant) }\end{array}$ & $\leftarrow$ & $\longrightarrow$
\end{tabular}

Figure 8 Schematic representation of direction of influence of lexical stress.

for classing postlexical gemination (RS) as a discrete process, and indeed the present study has provided evidence that relative prominence may produce relative RS, occurring whenever some degree of prominence arises, only somewhat weakly when general prosodic conditions are 'hostile', for example when the adjacent stress is not phonetically prominent. The fact that $\mathrm{RS}$ does occur (albeit in a phonetically weak form) away from phonetic prominence indicates the extent to which RS is a grammaticalised process of lengthening.

\section{Acoustic study of non-durational indices}

A subset of the material recorded for the first part of the investigation was also subject to a formant analysis. This subset consisted of Type 1 (word-internal, lexical) and Type 3 (wordboundary, productive RS) geminates and their non-geminate counterparts, for the consonant $/ 1 /$, in the vowel context /a/, for nuclear position only. /1/ was chosen because out of the consonants examined in this study, it presents the clearest formant structure. Lexical stress conditions were as set out in table 3 above.

The aim of the formant analysis was to test for the presence of gestural differences between geminates and non-geminates. A lower F1 and higher F2 and F3 were taken as an indication of a more palatalised configuration of the vocal tract. Support for this interpretation can be found in measurements reported by Vagges et al. (1979) for the Italian palatal lateral $/ \mathrm{K}: /$, clearly showing a lower F1 and higher F2 and F3 when compared with the alveolar lateral /1/. Comparison with $/ K: /$ does not of course mean that contact must be palatal; it is the OVERALL CONFIGURATION of the vocal tract that counts. Indeed, no contact has to occur at all for a configuration to be palatalised, as is the case with the vowel /i/. Since the tongue muscles are not wholly independent of one another, the overall configuration for an alveolar constriction will be more or less palatal, depending on how much contact is made AND on the overall shape of the tongue (i.e. is the whole of the tongue body generally convex in shape, or is there relative concavity behind the constriction?). A greater amount of contact will entail a 'flatter' configuration, with the tongue body more raised towards the palate. A smaller amount of contact is more likely to indicate an apical articulation, during which it is not uncommon for the tongue back to be 'cupped'.

\subsection{Analysis}

Frequency measurements were taken at the mid-point of the consonant for the first three formants. The waveform provided guidance for the positioning of these points on the spectrogram, while bandwidths were closely monitored to ensure that measurements taken corresponded to actual formants. Spectra were computed for a Hanning window of $30 \mathrm{~ms}$, set to search for 18 poles. To aid measurement and interpretation, reference was made to the values recorded for Italian /1/ by Ferrero et al. (1979) and Vagges et al. (1979). Separate multivariate repeated measures ANOVAs were run on measurements for F1, F2 and F3 for each condition. 


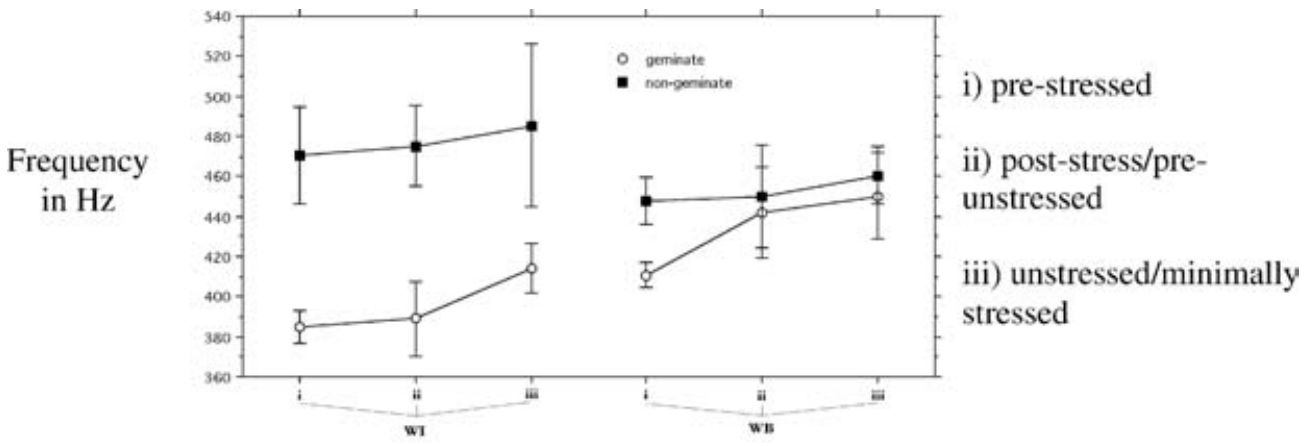

Position in the word and stress condition

Figure 9 Mean frequency of $\mathrm{F} 1$ in $/ 1 /$ and $/ 1:$ as a function of stress and position in word (error bars indicate confidence error bars of $95 \%$ ).

Table 25 Mean frequency in $\mathrm{Hz}$, and standard deviations, of $\mathrm{F} 1$ in $/ 1$ / and /l: / as a function of stress and position in word.

\begin{tabular}{llllll}
\hline & \multicolumn{2}{c}{ Word-internal } & & \multicolumn{2}{c}{ Word-boundary } \\
\cline { 2 - 3 } \cline { 6 - 7 } & Geminate F1 (sd) & Non-geminate F1 (sd) & & Geminate F1 (sd) & Non-geminate F1 (sd) \\
\hline Pre-stress & $383.333(19.906)$ & $472.233(30.598)$ & & $411.400(14.922)$ & $449.200(6.551)$ \\
Pre-unstressed & $386.133(55.415)$ & $478.933(27.986)$ & & $441.400(33.455)$ & $451.100(57.361)$ \\
Unstressed & $415.233(30.167)$ & $482.200(98.050)$ & & $454.467(65.608)$ & 459.267 (17.103) \\
\hline
\end{tabular}

\subsection{Results}

As figure 9 and table 25 show, F1 is consistently lower at the mid-point of the geminate than at the mid-point of the non-geminate, for all stress conditions. However, the difference is only statistically significant word-internally, where on average F1 is $81 \mathrm{~Hz}$ lower during the geminate than during the non-geminate (pre-stress, $\mathrm{F}(20,2)=19.443, \mathrm{P}=0.0027$; poststress, $\mathrm{F}(20,2)=28.298, \mathrm{P}=0.0026$; unstressed, $\mathrm{F}(20,2)=12.382, \mathrm{P}=0.0073)$. The mean difference at word boundaries is only $19 \mathrm{~Hz}$ and is only statistically significant in a prestress context $(\mathrm{F}(20,2)=4.661, \mathrm{P}=0.0329)$. $\mathrm{F} 1$ is lowest (consonant most palatal) when the consonant is pre-stress, for both word-internal and word-boundary position, and for both geminate and non-geminate consonants. The least palatal (highest F1) occurs when unstressed (word-internal) or minimally stressed (word-boundary). Interestingly, while F1 is lower in word-internal geminates than in equivalent word-boundary geminates, the reverse is true of non-geminates. As a result, the difference between geminates and non-geminates, as with duration, is greatest word-internally.

Though the picture for F2 is less consistent, where there is a discernible difference the trend is for F2 to be higher in geminates, as can be seen in figure 10 and table 26. Word-internally and at word boundaries, F2 is higher during geminates when pre-stress or unstressed/minimally unstressed, and the differences are statistically significant (wordinternal, $\mathrm{F}(20,1)=9.239, \mathrm{P}=0.0083 ; \mathrm{F}(20,1)=5.557, \mathrm{P}=0.0412$; word-boundary, $\mathrm{F}(20$, $1)=6.303, \mathrm{P}=0.0121 ; \mathrm{F}(20,1)=13.517, \mathrm{P}=0.0033)$. At word boundaries, $\mathrm{F} 2$ is higher during non-geminates in pre-unstressed position, and this is marginally significant $(\mathrm{F}(2$, $1)=2.784, \mathrm{P}=0.0578$ ). 


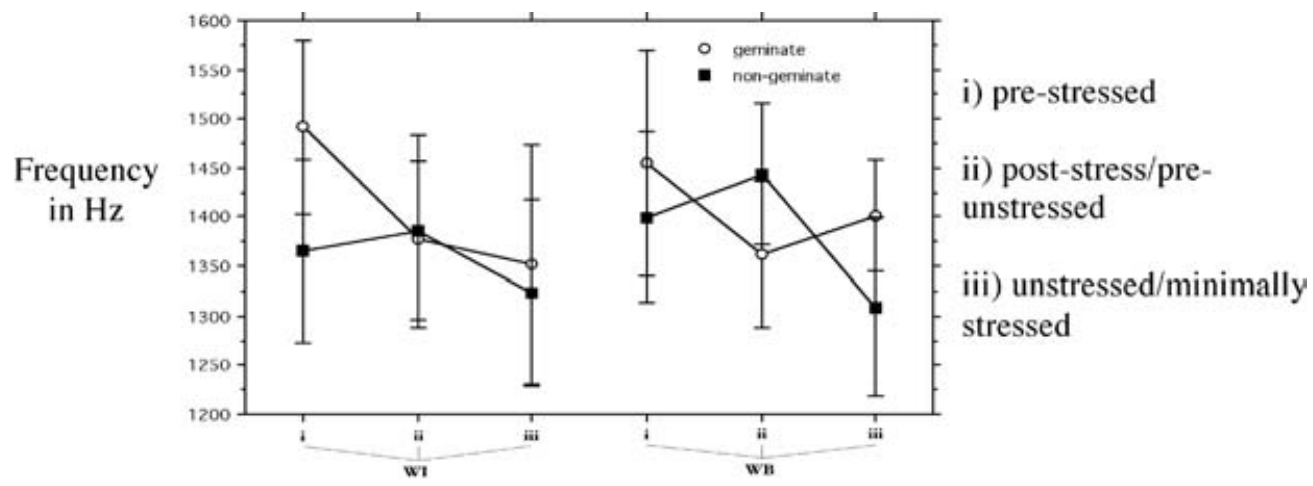

Position in the word and stress condition

Figure 10 Mean frequency of F2 in /1/ and /1:/ as a function of stress and position in word.

Table 26 Mean frequency in $\mathrm{Hz}$, and standard deviations, of $\mathrm{F} 2$ in /1 / and /1:/ as a function of stress and position in word.

\begin{tabular}{|c|c|c|c|c|}
\hline & \multicolumn{2}{|c|}{ Word-internal } & \multicolumn{2}{|c|}{ Word-boundary } \\
\hline & Geminate (sd) & Non-geminate (sd) & Geminate (sd) & Non-geminate (sd) \\
\hline Pre-stress & 1495.922 (222.053) & 1369.942 (225.546) & $1454.208(266.640)$ & 1403.381 (200.543) \\
\hline Pre-unstressed & $1374.033(237.432)$ & 1382.378 (158.998) & 1368.372 (199.408) & 1486.700 (167.340) \\
\hline Unstressed & $1361.442(294.164)$ & 1328.922 (135.729) & $1401.922(135.729)$ & $1310.228(184.236)$ \\
\hline
\end{tabular}

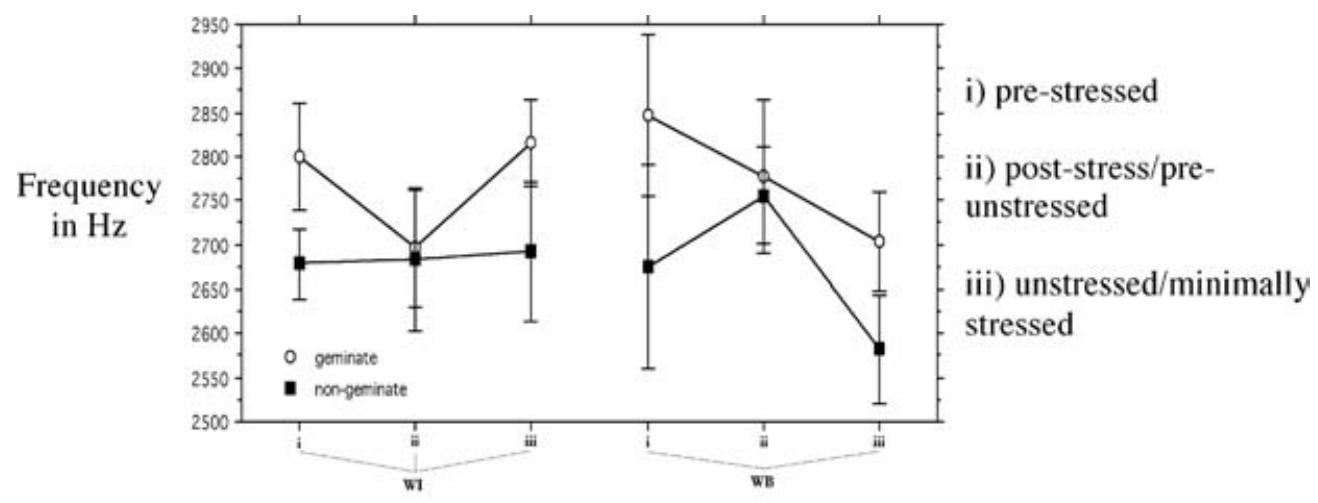

Position in the word and stress condition

Figure 11 Mean frequency of F3 in /1/ and /1:/ as a function of stress and position in word.

As figure 11 and table 27 show, F3 values are higher for geminates in all stress conditions. However, the differences are statistically significant only for pre-stress and unstressed/ minimally stressed position (word-internal, $\mathrm{F}(20,1)=4.544, \mathrm{P}=0.0360 ; \mathrm{F}(20,1)=5.612$, $\mathrm{P}=0.0342$; word-boundary, $\mathrm{F}(20,1)=7.231, \mathrm{P}=0.0072 ; \mathrm{F}(20,1)=4.111, \mathrm{P}=0.0346)$. 
Table 27 Mean frequency in $\mathrm{Hz}$, and standard deviations, of $\mathrm{F3}$ in /1 / and /1:/ as a function of stress and position in word.

\begin{tabular}{llllll}
\hline & \multicolumn{2}{c}{ Word-internal } & & \multicolumn{2}{c}{ Word-boundary } \\
\cline { 2 - 3 } & Geminate (sd) & Non-geminate (sd) & & Geminate (sd) & Non-geminate (sd) \\
\hline Pre-stress & $2809.115(297.125)$ & $2679.286(183.368)$ & & $2852.387(318.158)$ & $2679.286(317.578)$ \\
Pre-unstressed & $2703.816(265.492)$ & $2685.286(234.584)$ & & $2781.582(187.582)$ & $2756.284(212.276)$ \\
Unstressed & $2821.275(217.559)$ & $2692.296(207.286)$ & & $2711.692(245.188)$ & $2582.296(195.373)$ \\
\hline
\end{tabular}

This pattern echoes that for F2, suggesting the non-durational indices of the geminate/nongeminate contrast, are diminished in post-stress/pre-unstressed position. Interestingly, the durational contrast for $/ 1 /$ was also greatest in this position, unlike the majority of other consonant types for which contrast tended to be greater post-stress.

To summarise, geminate /1: is characterised by a lower F1 and (except for post-stress/preunstressed position) higher F2 and F3. This difference in formant structure is indicative of a gestural difference between geminates and non-geminates, with the articulation of /l:/ involving a more palatalized configuration than for /1/, particularly when word-internal and pre-stress or unstressed /minimally stressed. Comparing mean values for word-internal geminate $/ \mathrm{l}$ / with those for non-geminate $/ \mathrm{l} /$ and the palatal lateral $/ K /$ (which, like $/ \mathrm{J} /$, is conventionally classed as an inherently geminate), we see that the geminate /l: lies midway between the non-geminate and the (geminate) palatal lateral. These values are shown in figure 12. Formant values for $/ K /$ and a further set of values for $/ 1 /$ are taken from Vagges et al. 1979, with the caveat that the authors do not specify whether informants were male or female.

\subsection{Discussion}

The formant analysis points to a more palatalised vocal tract configuration in geminate laterals, indicating that the distinction of phonological length in Italian may not be phonetically interpreted through durational differences alone. At a practical level, non-durational acoustic cues may be of value in the perception of geminate contrasts: although such indices are varyingly robust and do not occur uniformly, the possibility remains that where they occur they supplement durational cues. The theoretical implications of non-durational indices are not easy to determine. From a gestural point of view, the more palatal configuration of the vocal tract during geminates may be interpreted in one of two ways. One possibility is that the difference is an unplanned result of extended duration. While it is assumed that a more palatal configuration is not universally an inevitable result of increased duration, it may be that the particular gestural strategy adopted to increase duration in this variety of Italian also happens to result in a more palatal configuration. The observed cline of palatalisation from $[1]>[1:]>[K:]$ does indeed suggest a phonetic correlation between palatalisation and longer duration in Italian. Alternatively, during the shorter non-geminate there may simply be less time for the articulators to reach their target, i.e. gestural undershoot. On this interpretation, the underlying gestural targets for geminates and non-geminates would essentially be the same, bar the temporal aspect, which could be specified by adding the feature [+long] or, in Articulatory Phonology (see e.g. Browman \& Goldstein 1992), adjusting gestural stiffness. 


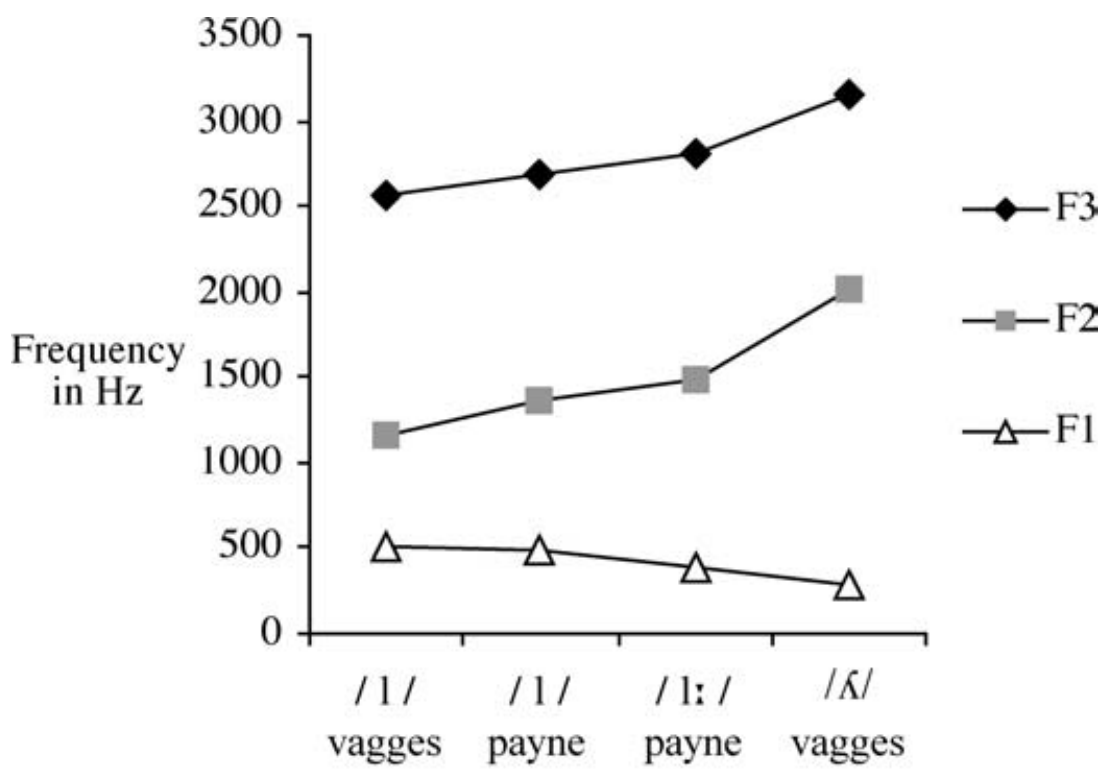

Type of lateral

Figure 12 Average values of the first three formants in Italian laterals.

If this were the case, we might expect the palatal effect to be more uniform, or at the very least be consistent with variation in duration, i.e. least strong in minimally stressed position, when geminates are at their phonetic shortest. However, this is not the case. Although F1 is always lower in the geminate, F2 and F3 are only higher for two out of three lexical stress conditions.

Another possibility is that the observed surface differences are due to a difference in the UNDERLYING representation of geminates and non-geminates, and therefore present at the stage of gestural planning. On this interpretation, the underlying gesture for $/ \mathrm{l} / \mathrm{w}$ would be SPATIALLY different from the gesture for $/ 1 /$. The fact that the effects are stronger in wordinternal geminates, particularly with respect to the low F1, might suggest that non-durational indices are a more robust feature of LEXICAL geminates, and that this type of geminate alone is gesturally different from non-geminates. However, again, if this were the case one would expect greater uniformity in the presence and absence of the palatalising effect. However, post-stress non-geminates also appear to be more palatalised. It may be that post-stress nongeminates (which, as has been shown, are subject to phonetic lengthening) are subject to a separate, phonetic palatal effect, thereby reducing the effective contrast with geminates in that position. On this interpretation, post-stress position could be interpreted as generally FORTIS. At word boundaries, non-geminates cannot be post-stress, and would therefore never be subject to this effect, and the distinction would not be undermined. How post-lexical geminates themselves acquire the palatal effect depends on how one views their structural status. If the transformation into geminate is interpreted as occurring at the level of gestural planning, the palatal effect will also be acquired at this stage; if gemination occurs as an 
add-on at the production stage, the palatal effect will presumably be acquired phonetically (and perhaps only as a result of being post-stress).

\section{Conclusion}

This study has revealed a high degree of plasticity in the phonetic interpretation of geminate consonants in Italian, with geminate and non-geminate duration and the contrast between these dependent on an interaction of consonant type, position in the word and prosodic factors. That some of these factors appear to mimic historically grammaticalised forms of gemination is particularly noteworthy, and may provide insight into the mechanisms by which sound systems evolve. With regard to prosodic conditioning, the phonetic tendency for post-stress lengthening (albeit only when that stress is intonationally prominent) mirrors the phonologised process of postlexical gemination. As for articulatory factors, the INTRINSIC plasticity according to consonant type in duration and the robustness of the geminate contrast appears to correlate with functional load. The evidence suggests that, historically, the various different formalisations of lengthened consonants into structurally significant geminates come about through the structural anchorage of low-level languagespecific phonetic properties, properties which are still present in the language. It should be borne in mind that lexical geminates, when not inherited directly from Latin, at times result from other phonetic processes, e.g. assimilation, which may also be prominencerelated.

In addition to prosodic and articulatory shaping, the study also presents evidence that geminate consonants may also be SPATIALLY distinct from non-geminates. Observed differences in the formant structure of laterals are not easy to explain mechanistically. For the purpose of underlying contrast, it is arguably of little import how, precisely, that contrast is signalled, and anyway by far the most salient and robust index is duration. Nevertheless, additional cues may be of supplementary value in perception, especially if the effects of fortition are long domain, as Local \& Simpson (1999) claim for Malayalam. Moreover, non-durational properties of geminates may underlie differences in the 'success' of individual consonant types and consequently be instrumental in historical processes.

\section{Acknowledgements}

The author wishes to thank the British Academy for the funding of a Postdoctoral Fellowship at the Department of Linguistics, University of Cambridge, Pier Marco Bertinetto for use of the Linguistics Laboratory at the Scuola Normale di Pisa, and Francis Nolan, Edda Farnetani, Peter Ladefoged, John Esling and an anonymous JIPA referee for their helpful comments on this paper.

\section{References}

ABramson, A. S. (1991). Amplitude as a cue to word-initial consonant length: Pattani Malay. In Proceedings of the 12th International Congress of Phonetic Sciences, Aix-en-Provence 1, 98-101. 
Abramson, A. S. (1999). Fundamental Frequency as a cue to word-initial consonant length: Pattani Malay. In Proceedings of the 14th International Congress of Phonetic Sciences, Berkeley, University of California 1, 591-594.

AgOSTINIANI, L. (1992). Su alcuni aspetti del 'rafforzamento sintattico' in Toscana e sulla loro importanza per la qualificazione del fenomeno in generale. Quaderni del Dipartimento di Linguistica dell' Università degli Studi, University of Florence 3, 1-28.

ARVANITI, A. (1999). Effects of speaking rate on the timing of single and geminate sonorants. In Proceedings of the 14th International Congress of Phonetics Sciences, Berkeley, University of California 1, 595-598.

BORTOLINI, U. \& ZAMPOLLI, A. (1979). Frequenza e distribuzione dei gruppi consonantici nella lingua italiana. Acta Phoniatrica 1, 195-208.

Browman, C. \& GoldsteIn, L. (1992). Articulatory phonology: an overview. Phonetica 49, $155-80$.

CANEPARI, L. (1999). Manuale di pronuncia italiana (MaPI). Bologna: Zanichelli.

CoHN, A. C., HAM, W. H. \& PODEsva, R. J. (1999). The phonetic realisation of singleton-geminate contrasts in three languages of Indonesia. In Proceedings of the 14th International Congress of Phonetic Sciences, Berkeley, University of California 1, 587-590.

ERICSDOTTER, C. (1998). Effects of stress and speaking rate on the Italian geminate contrast: durational measurements. Conference paper at Fonetik 98, Stockholm.

FANCIULlO, F. (1986). Syntactic reduplication and the Italian dialects of the Centre-South. Journal of Italian Linguistics 8, 67-104.

Ferrero, F. E., Magno-Caldognetto, E., Vagges, K. \& Lavagnoli, C. (1979). Some Acoustic characteristics of the Italian vowels. Journal of Italian Linguistics 4, 87-95.

KEATING, P. A., WRIGHT, R. \& ZHANG, J. (1999). Word-level asymmetries in consonant articulation. UCLA Working Papers in Phonetics 97, 157-173.

KLATT, D. N. (1976). Linguistic uses of segmental duration in English: acoustic and perceptual evidence. Journal of Acoustical Society of America 59, 1208-1221.

LADD, D. R. \& ScobBIE, J. M. (2003). External sandhi as gestural overlap? Counter-evidence from Sardinian. In Local, J., Ogden, R. \& Temple, R. (eds.), Papers in Laboratory Phonology VI: Phonetic Interpretation. Cambridge: Cambridge University Press.

LeHISTE, I. (1970). Suprasegmentals. Cambridge, MA: MIT Press.

LOCAL, J. \& SiMPSON, A. (1999). Phonetic implementation of geminates in Malayalam nouns. In Proceedings of the 14th International Congress of Phonetic Sciences, Berkeley, University of California 1, 599-612.

LOPORCARO, M. (1997). L'origine del raddoppiamento fonosintattico. Basel: Francke Verlag.

LOUALI, N. \& MADDIESON, I. (1999). Phonological contrast and phonetic realisation: the case of Berber stops. In Proceedings of the 14th International Congress of Phonetic Sciences, Berkeley, University of California 1, 603-606.

NeSPOR, M. \& Vogel, I. (1986). Prosodic Phonology. Dordrecht: Foris Publications.

MAIDEN, M. \& PARRY, M. (1997). The dialects of Italy. London: Routledge.

MARotTA, G. (1986). Rhythmical constraints on syntactic doubling. Journal of Italian Linguistics 8 , $35-52$.

PAYNe, E. M. (2000). Consonant Gemination in Italian: Phonetic Evidence for a Fortition Continuum. $\mathrm{Ph} . \mathrm{D}$. thesis, University of Cambridge.

Rogers, D. \& D'ArCAngeli, L. (2004). Italian. Journal of International Phonetic Association 34, 117 121.

Vagges, K., Ferrero, F. E., Magno-Caldognetto, E. \& Lavagnoli, C. (1979). Some acoustic characteristics of Italian consonants. Journal of Italian Linguistics 3-4, 69-85. 
180 E. M. Payne

\section{Appendix: Target words used}

Table A1 Word-internal target words.

\begin{tabular}{|c|c|c|c|c|c|c|}
\hline & & Geminate & & & Non-geminat & \\
\hline & Pre-stress & Post-stress & Unstressed & Pre-stress & Post-stress & Unstressed \\
\hline$/ \mathrm{p} /$ & pappato & рарра & pappagallo & papale & Рapa & capannina \\
\hline & wolfed down & mush & parrot & papal & Pope & cabin \\
\hline$/ \mathrm{b} /$ & sabbatico & gabba & gabbamondo & rabarbaro & sabato & tabaccaio \\
\hline & sabbatical & cage & swindler & rhubarb & Saturday & tobacconist \\
\hline$/ \mathrm{t} /$ & fattori & fatto & fattorino & batosta & dato & patologico \\
\hline & factors & done & postman & blow & given & pathological \\
\hline$/ \mathrm{d} /$ & addice & caddi & additare & adito & cadi & adibito \\
\hline & it suits & I fell & to point at & taken & you fall & assigned \\
\hline$/ \mathrm{f} /$ & affabile & gaffa & affamato & safari & rafani & Rafanello \\
\hline & affable & boat hook & starving & safari & radishes & (proper name) \\
\hline$/ 1 /$ & ballato & balla & ballatoio & calata & cala & palazzina \\
\hline & danced & s/he dances & gallery & subsided & it subsides & villa \\
\hline$/ \mathrm{m} /$ & rammaglio & mamma & rammagliare & affamati & fama & amaretto \\
\hline & I darn & mum & to darn & starved (pl) & fame & type of liqueur \\
\hline
\end{tabular}

Table A2 Word-boundary target words.

\begin{tabular}{|c|c|c|c|c|c|c|}
\hline & \multicolumn{3}{|c|}{ Geminate } & \multicolumn{3}{|c|}{ Non-geminate } \\
\hline & Pre-stress & Pre-unstressed & Minimally stressed & Pre-stress & Pre-unstressed & Minimally stressed \\
\hline$/ \mathrm{p} /$ & diventerà Papa & sarà pappato & diventerà panettiere & diventa Papa & corona papale & diventa panettiere \\
\hline & he'll become Pope & he'll have wolfed down & s/he'll become a baker & he becomes Pope & papal crown & s/he becomes a baker \\
\hline$/ \mathrm{b} /$ & dirà balle & avrà ballato & farà balbettare & racconta balle & ama ballare & diventa balbettante \\
\hline & s/he'll tell lies & s/he'll have danced & it'll make (someone) stutter & s/he tells lies & s/he loves to dance & s/he becomes stuttering \\
\hline$/ \mathrm{t} /$ & $\begin{array}{l}\text { mangerà tonno } \\
\text { s/he'll eat tuna }\end{array}$ & $\begin{array}{l}\text { sarà toccata } \\
\text { she/it'll be touched }\end{array}$ & $\begin{array}{l}\text { sarà Topolino } \\
\text { it'll be Mickey Mouse }\end{array}$ & $\begin{array}{l}\text { mangia tonno } \\
\text { s/he eats tuna }\end{array}$ & $\begin{array}{l}\text { la toccare } \\
\text { to touch her/it }\end{array}$ & $\begin{array}{l}\text { ama Topolino } \\
\text { s/he loves Mickey Mouse }\end{array}$ \\
\hline$/ \mathrm{d} /$ & $\begin{array}{l}\text { comprerà ditte } \\
\text { s/he'll buy firms }\end{array}$ & $\begin{array}{l}\text { città dinamica } \\
\text { dynamic city }\end{array}$ & $\begin{array}{l}\text { sarà diligente } \\
\text { s/he'll be diligent }\end{array}$ & $\begin{array}{l}\text { compra ditte } \\
\text { s/he buys firms }\end{array}$ & $\begin{array}{l}\text { ditta dinamica } \\
\text { dynamic firm }\end{array}$ & $\begin{array}{l}\text { sembra diligente } \\
\text { s/he seems diligent }\end{array}$ \\
\hline$/ \mathrm{f} /$ & $\begin{array}{l}\text { Diventerà facile } \\
\text { it'll become easy }\end{array}$ & $\begin{array}{l}\text { diventerà fanatico } \\
\text { he'll become fanatical }\end{array}$ & $\begin{array}{l}\text { città facoltosa } \\
\text { rich city }\end{array}$ & $\begin{array}{l}\text { diventa facile } \\
\text { it becomes easy }\end{array}$ & $\begin{array}{l}\text { diventa fanatico } \\
\text { he becomes fanatical }\end{array}$ & $\begin{array}{l}\text { ditta facoltosa } \\
\text { rich firm }\end{array}$ \\
\hline$/ 1 /$ & mangerà l'anitra & mangerà lasagne & diventerà latitante & mangia l'anitra & mangia lasagne & diventa latitante \\
\hline$/ \mathrm{m} /$ & $\begin{array}{l}\text { s/he'll eat duck } \\
\text { diventerà matto } \\
\text { he'll go mad }\end{array}$ & $\begin{array}{l}\text { s/he'll eat lasagne } \\
\text { diventerà malato } \\
\text { he'll get ill }\end{array}$ & $\begin{array}{l}\text { s/he'll become a fugitive } \\
\text { mangerà maccheroni } \\
\text { s/he'll eat maccheroni }\end{array}$ & $\begin{array}{l}\text { s/he eats duck } \\
\text { diventa matto } \\
\text { he gets mad }\end{array}$ & $\begin{array}{l}\text { s/he eats lasage } \\
\text { diventa malato } \\
\text { he gets ill }\end{array}$ & $\begin{array}{l}\text { s/he becomes a fugitive } \\
\text { mangia maccheroni } \\
\text { s/he eats maccheroni }\end{array}$ \\
\hline
\end{tabular}


Table A3 / / t target words.

\begin{tabular}{|c|c|c|c|c|c|}
\hline & \multicolumn{4}{|c|}{ Word-boundary } & \multirow{2}{*}{$\begin{array}{l}\text { False } \\
\text { C-C }\end{array}$} \\
\hline & $\begin{array}{l}\text { Word-internal } \\
\text { VCV }\end{array}$ & C-CV & V-CV & 'V-CV & \\
\hline \multirow[t]{2}{*}{ Pre-stress } & lasciare & non sciarpe & compra sciarpe & comprerà sciarpe & flash scialbo \\
\hline & to leave & not shoes & s/he buys shoes & s/he will buy shoes & pale flash \\
\hline \multirow[t]{2}{*}{ Pre-unstressed } & lascia & non sciaquare & deva sciacquare & dovrà sciaquare & smash sciancante \\
\hline & s/he leaves & don't rinse & (that) you must rinse & s/he will have to rinse & crippling smash \\
\hline \multirow[t]{2}{*}{ Maximally unstressed } & lasciavamo & non sciammanare & pratica sciacallaggio & praticherà sciacallaggio & crash sciagurato \\
\hline & we used to leave & don't untidy & s/he practices exploitation & s/he'll practice exploitation & unlucky crash \\
\hline
\end{tabular}

Table A4 False geminate target words.

\begin{tabular}{llll}
\hline & \multicolumn{3}{c}{ Geminate } \\
\cline { 2 - 4 } & Pre-stress & Pre-unstressed & Maximally unstressed \\
\hline$/ \mathrm{p} /$ & gap pazzo & gap palese & gap passeggero \\
& crazy gap & evident gap & transient gap \\
/t/ & Fiat Tipo & Fiat Ticino & Fiat Ticinese \\
& (make of car) & (make of car) & Fiat from Ticino \\
$/ \mathbf{f} /$ & gaffe facile & gaffe fatale & gaffe favolosa \\
& easy gaffe & fatal gaffe & fabulous gaffe \\
$/ \mathbf{l} /$ & al lato & al lattaio & al latitante \\
& to the side & to the dairyman & to the fugitive \\
$/ \mathbf{m} /$ & Sam Mantova & Sam Mattei & Sam Mantovani \\
& (proper name) & (proper name) & (proper name) \\
\hline
\end{tabular}

